\title{
A Hybridizable Discontinuous Galerkin phase-field model for brittle fracture with adaptive refinement
}

\author{
Alba Muixí | Antonio Rodríguez-Ferran I Sonia Fernández-Méndez*
}

${ }^{1}$ Laboratori de Càlcul Numèric (LaCàN), Universitat Politècnica de Catalunya, Barcelona 08034, Spain

Correspondence

*Sonia Fernández-Méndez. Email: sonia.fernandez@upc.edu

\begin{abstract}
In this paper, we propose an adaptive refinement strategy for phase-field models of brittle fracture, which is based on a novel Hybridizable Discontinuous Galerkin (HDG) formulation of the problem. The adaptive procedure considers standard elements and only one type of $h$-refined elements, dynamically located along the propagating cracks. Thanks to the weak imposition of inter-element continuity in HDG methods, and in contrast with other existing adaptive approaches, hanging nodes or special transition elements are not needed, which simplifies the implementation. Various numerical experiments, including one branching test, show the accuracy, robustness and applicability of the presented approach to quasi-static phase-field simulations.

KEYWORDS:

phase-field modeling, brittle fracture, Hybridizable Discontinuous Galerkin (HDG), staggered scheme, adaptive refinement
\end{abstract}

\section{1 | INTRODUCTION}

Phase-field models of fracture are very demanding on the spatial discretization: very fine meshes are required to capture the solutions with narrow damage bands typical of small length-scale parameters $\frac{1213445}{\text {. }}$.

In some problems, the crack path is known in advance, either exactly or approximately; consider, for instance, a straight crack in a symmetrical quasi-static configuration or a curved crack starting at a notch tip. In such scenarios, one may resort to a non-structured mesh with small elements along the expected crack path and larger elements elsewhere. This is a rather common approach $12 / 45$. On the contrary, if the crack path is not known beforehand, as for example happens in branching tests in dynamic fracture, or with complex crack patterns in heterogenous media, an adaptive strategy is needed. In such approaches, the discretization is dynamically refined along cracks as they propagate. Some recent proposals on this direction are discussed next.

Nagaraja et al ${ }^{6]}$ present a continuous approach based on the multi-level $h p$-FEM and the Finite Cell Method. For a fixed polynomial degree $p$, the mesh is $h$-refined recursively up to depth $k$, as dictated by the damage field, with a geometrical grading from the coarse initial mesh to the finest mesh. This multi-level refinement relies on the use of regular grids; to handle irregular boundaries, it is combined with the Finite Cell Method, an immersed boundary approach.

Patil et al ${ }^{7}$ propose an adaptive continuous-discontinuous approach inspired by the Xfield method by Giovanardi et al ${ }^{8}$. The phase-field equations are only solved in an adaptive circular window centred in the crack tip; outside that region, the crack is sharply represented via the X-FEM enrichment of the displacement field. Mesh adaptivity also relies on the multi-level FEM, which provides a transition between the very fine mesh near the crack and the coarse mesh away from it.

The above mentioned strategies deal with hanging nodes and impose continuity between elements in strong form. 


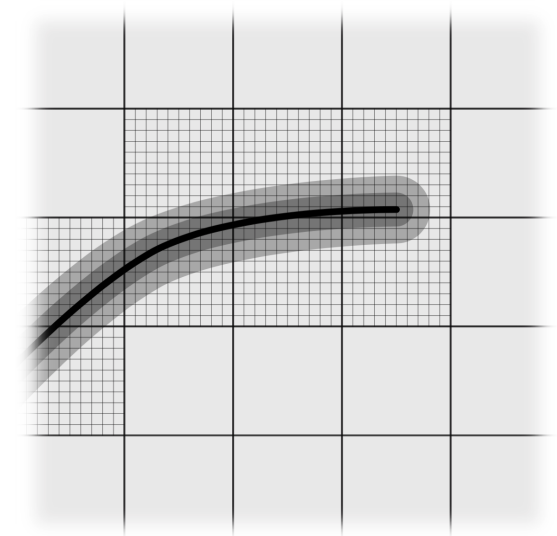

FIGURE 1 Illustration of the proposed adaptive discretization: refined elements along the crack, where the phase-field variable exceeds a threshold value, and standard elements in the rest of the domain.

Noii et a ${ }^{19}$ use a Global-Local method: they consider independent meshes, for the global domain and for the region along the crack. Continuity between meshes is weakly imposed using Lagrange multipliers, which are added as new unknowns to the problem. The refinement is driven by a predictor-corrector scheme ${ }^{10}$. Adaptive methodologies for brittle fracture have also been proposed in the framework of isogeometric analysis 11112 .

The main goal of this paper is to propose an adaptive strategy for phase-field models based on an element-by-element local refinement along crack paths, without refinement transition regions nor additional unknown fields.

The strategy is based on imposing the continuity of the displacement and damage fields between elements in weak form, in the setting of a Discontinuous Galerkin spatial discretization. Only two types of elements are considered in our approach: standard elements and refined elements. Each type of element is mapped to the corresponding reference element (standard or refined), and the original background mesh is kept fixed during all the simulation. The refined reference element is uniformly split in subelements, with the corresponding basis functions and integration points. This simplifies the implementation, reducing to the minimum the cases and the computational cost of the adaptive process. The very local and non-conformal $h$-refinement along the crack is illustrated in Figure 1. The level of refinement for the elements along the crack is chosen according to the lengthscale parameter of the model. A video showing the adaptive refinement for a branching test, included in the numerical examples, is available in YouTube ${ }^{13}$.

Within the DG family, we choose a Hybridizable Discontinuous Galerkin (HDG) method, because they are significantly cheaper than other DG methods, with a computational efficiency close to Continuous Galerkin (CG) methods $\frac{[14|15| 16 / 17 / 18}{\text {. The }}$ adaptive strategy is tested with the hybrid phase-field model of Ambati et $\mathrm{al}^{1}$, but it is also applicable to other models.

The paper is structured as follows. Section 2 briefly reviews the hybrid phase-field model. A novel HDG formulation for its solution is developed in Section 3 The proposed adaptive strategy is discussed in detail in Section 4 Numerical examples in Section 5 demonstrate the applicability and robustness of the methodology, providing accurate solutions with a very localized refinement along cracks and no transition regions. The concluding remarks of Section 6 close the paper.

\section{2 | HYBRID PHASE-FIELD MODEL FOR FRACTURE}

In this section we give an overview of the hybrid phase-field model for quasi-static brittle fracture proposed by Ambati et al ${ }^{1}$. Within a staggered implementation, this model leads to linear subproblems while considering a tension-compression splitting of the elastic energy to avoid cracking in compression.

Consider an elastic body occupying a domain $\Omega \subset \mathbb{R}^{n_{s d}}$, with $n_{s d}$ the spatial dimension, under the hypothesis of small deformations and with a traction-free crack denoted by $\Gamma_{C}$, see Figure 2 (left). Let $\boldsymbol{u}(\boldsymbol{x}, t)$ be the displacement vector at a point $x \in \Omega$ at time $t$. The displacement field satisfies Dirichlet and Neumann boundary conditions on $\Gamma_{D}$ and $\Gamma_{N}$, respectively, with $\Gamma_{D} \cup \Gamma_{N}=\partial \Omega$ and $\Gamma_{D} \cap \Gamma_{N}=\emptyset$. The standard infinitesimal strain tensor is defined as $\varepsilon(\boldsymbol{u})=\left(\boldsymbol{\nabla} \boldsymbol{u}+(\nabla \boldsymbol{u})^{T}\right) / 2$. 
Following Francfort and Marigo ${ }^{19}$, the total energy of the body can be expressed as the sum of the bulk elastic energy and the crack surface energy, this is

$$
E\left(\boldsymbol{u}, \Gamma_{C}\right)=\int_{\Omega} \Psi_{0}(\varepsilon) \mathrm{d} V+G_{C} \int_{\Gamma_{C}} \mathrm{~d} s,
$$

with $\Psi_{0}$ the elastic energy density and $G_{C}$ the critical energy release rate. Minimization of the energy functional (1) determines the fracture process.

To enable the numerical treatment of (1), Bourdin et al ${ }^{[2}$ regularized the formulation by introducing a variable $d(\boldsymbol{x}, t)$, called the phase-field or damage parameter, smearing the representation of the crack, see Figure 2(right). The phase-field $d$ has value 0 at intact points of the material and 1 at fully damaged points, and varies smoothly between both values. The energy functional is approximated by

$$
E_{l}(\boldsymbol{u}, d)=\int_{\Omega}\left((1-d)^{2}+\eta\right) \Psi_{0}(\varepsilon) \mathrm{d} V+G_{C} \int_{\Omega}\left(\frac{d^{2}}{2 l}+\frac{l}{2}|\nabla d|^{2}\right) \mathrm{d} V,
$$

where $l$ is a length-scale parameter regulating the width of the smeared crack and $\eta$ is a small dimensionless parameter added to avoid a complete loss of stiffness in broken regions. This regularized formulation $\Gamma$-converges to the original one as $l$ tends to zero ${ }^{20}$. We restrict ourselves to the case of linear elastic isotropic materials, for which the elastic energy density is given by $\Psi_{0}(\varepsilon)=(\varepsilon: C: \varepsilon) / 2$, with $\boldsymbol{C}$ the fourth-order elasticity tensor depending on the Lamé parameters $\lambda$ and $\mu$.

Minimizing the regularized functional in (2) we obtain the governing equations

$$
\left\{\begin{array}{l}
\boldsymbol{\nabla} \cdot \boldsymbol{\sigma}=\mathbf{0} \\
-l^{2} \Delta d+d=\frac{2 l}{G_{C}}(1-d) \Psi_{0}
\end{array}\right.
$$

with the stress tensor $\sigma$ defined as

$$
\boldsymbol{\sigma}(\boldsymbol{u}, d)=\left((1-d)^{2}+\eta\right) \frac{\partial \Psi_{0}(\varepsilon)}{\partial \boldsymbol{\varepsilon}}=\left((1-d)^{2}+\eta\right) \boldsymbol{C}: \varepsilon(\boldsymbol{u}) .
$$

This formulation does not distinguish between tension and compression, and unphysical cracks under compression can appear ${ }^{2}$. Miehe et al ${ }^{\sqrt[314]{4}}$ proposed a splitting of the elastic energy density into its tensile and compressive components, $\Psi_{0}^{+}$and $\Psi_{0}^{-}$, based on the spectral decomposition of the strain tensor. More specifically, denoting by $\left\{\varepsilon_{i}\right\}_{i=1, \ldots, n_{s d}}$ the principal strains and by $\left\{\boldsymbol{n}_{i}\right\}_{i=1, \ldots, n_{s d}}$ the principal strain directions,

$$
\Psi_{0}^{ \pm}(\varepsilon)=\frac{1}{2} \lambda\langle\operatorname{tr}(\varepsilon)\rangle_{ \pm}^{2}+\mu \operatorname{tr}\left(\varepsilon_{ \pm}^{2}\right),
$$

where $\varepsilon_{ \pm}=\sum_{i=1}^{n_{s d}}\left\langle\varepsilon_{i}\right\rangle_{ \pm} \boldsymbol{n}_{i} \otimes \boldsymbol{n}_{i}$ and $\langle\odot\rangle_{ \pm}=(\odot \pm|\odot|) / 2$. The total energy functional is then redefined to allow only the degradation of the tensile energy $\Psi_{0}^{+}$,

$$
E_{l}(\boldsymbol{u}, d)=\int_{\Omega}\left(\left((1-d)^{2}+\eta\right) \Psi_{0}^{+}(\varepsilon)+\Psi_{0}^{-}\right) \mathrm{d} V+G_{C} \int_{\Omega}\left(\frac{d^{2}}{2 l}+\frac{l}{2}|\nabla d|^{2}\right) \mathrm{d} V .
$$
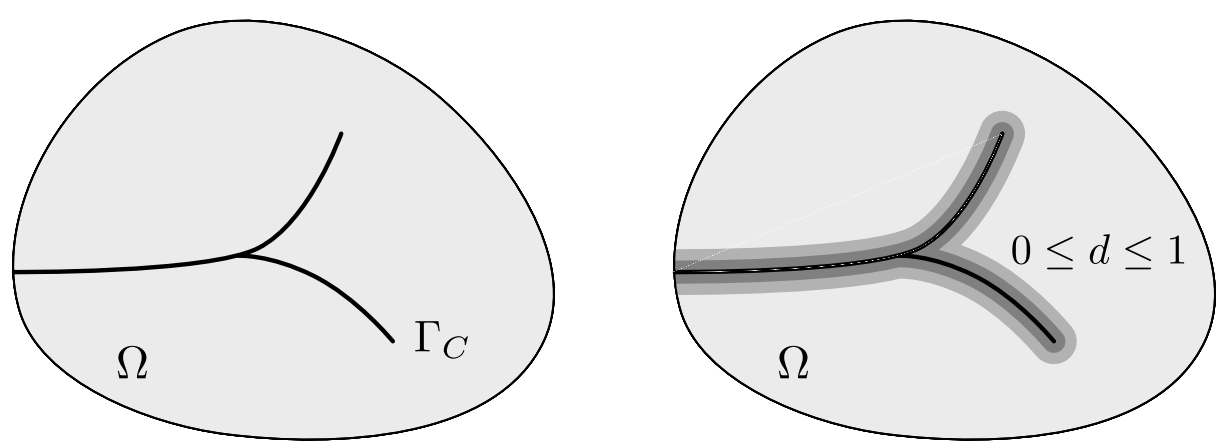

FIGURE 2 Left: Body with a sharp crack $\Gamma_{C}$. Right: Smeared crack representation. 
The stress-strain constitutive equation obtained in this case is

$$
\boldsymbol{\sigma}(\boldsymbol{u}, d)=\left((1-d)^{2}+\eta\right) \frac{\partial \Psi_{0}^{+}(\varepsilon)}{\partial \varepsilon}+\frac{\partial \Psi_{0}^{-}(\varepsilon)}{\partial \varepsilon},
$$

and the equation governing the phase-field evolution becomes

$$
-l^{2} \Delta d+d=\frac{2 l}{G_{C}}(1-d) \Psi_{0}^{+} .
$$

Notice in (8) that the phase-field variable only evolves due to tensile elastic energy, avoiding damage in compression. Also, keeping $\Psi_{0}^{-}$undegraded in (7) prevents the interpenetration of faces in case of crack closure.

To enforce irreversibility of cracks, Miehe et al ${ }^{3 / 4}$ replace $\Psi_{0}^{+}$in $(8)$ by a history-field variable defined as

$$
\mathcal{H}^{+}(\boldsymbol{x}, t)=\max _{\tau \in[0, t]} \Psi_{0}^{+}(\varepsilon(\boldsymbol{x}, \tau)) .
$$

Computationally, the main advantage of the isotropic model (3) is that both equations are linear within a staggered approach, while in formulations with a tension-compression splitting, the equilibrium equation becomes nonlinear due to the splitting in (7). In order to preserve the linearity of the equilibrium equation and to inhibit cracking in compression, Ambati et al ${ }^{11}$ proposed the so-called hybrid phase-field model, given by

$$
\left\{\begin{array}{l}
\boldsymbol{\nabla} \cdot \boldsymbol{\sigma}=\mathbf{0} \text { with } \boldsymbol{\sigma}=\left((1-d)^{2}+\eta\right) \frac{\partial \Psi_{0}(\varepsilon)}{\partial \boldsymbol{\varepsilon}}, \\
-l^{2} \Delta d+d=\frac{2 l}{G_{C}}(1-d) \mathcal{H}^{+}, \\
d:=0 \text { if } \Psi_{0}^{+}<\Psi_{0}^{-} .
\end{array}\right.
$$

Condition (10c) is added to the system to avoid interpenetration of faces, which otherwise is a possible scenario due to the stress-strain relation in 10a). This condition is not considered in this paper since it does not imply any changes under tensile stresses and, in our experience, diminishes the convergence of the staggered scheme. Because of this, we obtain a slight face interpenetration in the fourth numerical example in Section 5 For more details regarding the hybrid model we refer to Ambati et $\mathrm{al}^{1}$.

The system of equations (10) is solved in an incremental loading process. Assuming the solution at load step $n$ is known, the system is solved for the load step $n+1$ with the corresponding boundary conditions, that is,

$$
\begin{cases}\boldsymbol{\sigma} \cdot \boldsymbol{n}=\boldsymbol{t}^{n+1} & \text { on } \Gamma_{N}, \\ \boldsymbol{u}=\boldsymbol{u}_{D}^{n+1} & \text { on } \Gamma_{D}, \\ \boldsymbol{\nabla} d \cdot \boldsymbol{n}=0 & \text { on } \partial \Omega\end{cases}
$$

where $\boldsymbol{t}^{n+1}$ and $\boldsymbol{u}_{D}^{n+1}$ are the prescribed tractions and displacements at load step $n+1$, respectively, and $\boldsymbol{n}$ is the outward unit normal vector.

The phase-field model above is based on $i$ ) a spectral decomposition of the strain energy into tensile and compressive parts, equation (5); ii) a quadratic energetic degradation function $g(d)=(1-d)^{2}$, first integral in equation (6); iii) a quadratic geometric crack function $\alpha(d)=d^{2} / 2 l$, second integral in equation (6). Various alternative phase-field models, with different choices

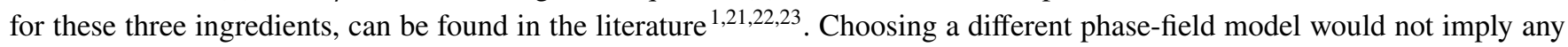
changes in the HDG adaptive strategy presented in this paper.

\section{3 | HDG FORMULATION}

Within a staggered approach to solve the system, we consider independent HDG formulations for the equilibrium and for the damage field equations. For the equilibrium equation, we choose the HDG formulation for linear elasticity proposed by Soon et $\mathrm{al}^{24}$, adding the damage degradation function in the stress-strain constitutive equation. However, the proposed strategy could also be applied with alternative HDG formulations in the literature ${ }^{25126}$. For the damage equation, we add the reaction term to the HDG formulation for diffusion by Cockburn et al ${ }^{27}$. In this section, we briefly recall both formulations and present the algorithm used to solve the coupled system of equations $(3$. 
In what follows, the domain $\Omega$ is covered by a finite element mesh with $\mathrm{n}_{\mathrm{el}}$ disjoint elements $K_{i}$ satisfying

$$
\bar{\Omega} \subset \bigcup_{i=1}^{\mathrm{n}_{\mathrm{el}}} \bar{K}_{i}, K_{i} \cap K_{j}=\emptyset \text { for } i \neq j,
$$

and the union of the $\mathrm{n}_{\mathrm{fc}}$ faces $\Gamma_{f}$ of the mesh is denoted as

$$
\Gamma=\bigcup_{i=1}^{\mathrm{n}_{\mathrm{el}}} \partial K_{i}=\bigcup_{f=1}^{\mathrm{n}_{\mathrm{fc}}} \Gamma_{f} .
$$

\subsection{HDG for the equilibrium equation}

Consider the equilibrium equation (10a) for a given damage field $d$. In the broken space of elements, the problem can be expressed as a set of local problems, one for each element, and some global equations on the skeleton of the mesh, $\Gamma$. Local problems state the equilibrium equation at each element $K_{i}$ with Dirichlet boundary conditions, this is,

$$
\left\{\begin{array}{lr}
\boldsymbol{\nabla} \cdot \boldsymbol{\sigma}(\boldsymbol{J}, d)=\mathbf{0} & \text { in } K_{i}, \\
\boldsymbol{J}-\boldsymbol{\nabla} \boldsymbol{u}=\mathbf{0} & \text { in } K_{i}, \\
\boldsymbol{u}=\hat{\boldsymbol{u}} & \text { on } \partial K_{i},
\end{array}\right.
$$

for $i=1 \ldots \mathrm{n}_{\mathrm{el}}$, with $\boldsymbol{\sigma}(\boldsymbol{J}, d)=\left((1-d)^{2}+\eta\right) \boldsymbol{C}: \frac{1}{2}\left(\boldsymbol{J}+\boldsymbol{J}^{T}\right)$. The new variable $\boldsymbol{J}$ is the gradient of $\boldsymbol{u}$, enabling to split the problem into a system of first order PDE, and $\hat{\boldsymbol{u}}$ is the trace of $\boldsymbol{u}$ on $\Gamma$, see Figure 3 Instead of introducing $\boldsymbol{J}$ as the mixed variable, one could use the strain tensor $\varepsilon$; both formulations are equivalent as proved by Fu et al 28 .

Given $\hat{\boldsymbol{u}}$, the local problems (12) can be solved to determine $\boldsymbol{u}$ and $\boldsymbol{J}$. Thus, the problem reduces to determining the trace variable $\hat{\boldsymbol{u}}$. This is done by solving the so-called global problem, which imposes equilibrium of tractions on faces and the boundary conditions, namely,

$$
\left\{\begin{array}{lr}
\llbracket \boldsymbol{\sigma} \cdot \boldsymbol{n} \rrbracket=\mathbf{0} & \text { in } \Gamma \backslash \partial \Omega, \\
\boldsymbol{\sigma} \cdot \boldsymbol{n}=\boldsymbol{t}_{N} & \text { on } \Gamma_{N}, \\
\hat{\boldsymbol{u}}=\boldsymbol{u}_{D} & \text { on } \Gamma_{D},
\end{array}\right.
$$

where $\llbracket \cdot \rrbracket$ stands for the jump operator defined at a face $\Gamma_{f}$ as $\llbracket \odot \rrbracket=\odot_{L_{f}}+\odot_{R_{f}}$, with $L_{f}$ and $R_{f}$ the left and right elements sharing the face and $\odot_{i}$ the value of $\odot$ from element $K_{i}$. Notice that the continuity of $\boldsymbol{u}$ across $\Gamma$ is imposed through the boundary condition $\boldsymbol{u}=\hat{\boldsymbol{u}}$ of the local problems, since $\hat{\boldsymbol{u}}$ is single-valued on faces.

The HDG formulation of the problem is obtained by discretizing the local and global problems. The discrete spaces considered to approximate the elemental variables, $\boldsymbol{u}$ and $\boldsymbol{J}$, and the trace variable, $\hat{\boldsymbol{u}}$, are

$$
\begin{aligned}
& \mathcal{V}^{h}(\Omega)=\left\{v \in L^{2}(\Omega):\left.v\right|_{K_{i}} \in \mathcal{P}^{p}\left(K_{i}\right) \text { for } i=1 \ldots \mathrm{n}_{\mathrm{el}}\right\}, \\
& \Lambda^{h}(\Gamma)=\left\{\hat{v} \in L^{2}(\Gamma):\left.\hat{v}\right|_{\Gamma_{f}} \in \mathcal{P}^{p}\left(\Gamma_{f}\right) \text { for } f=1 \ldots \mathrm{n}_{\mathrm{fc}}\right\},
\end{aligned}
$$
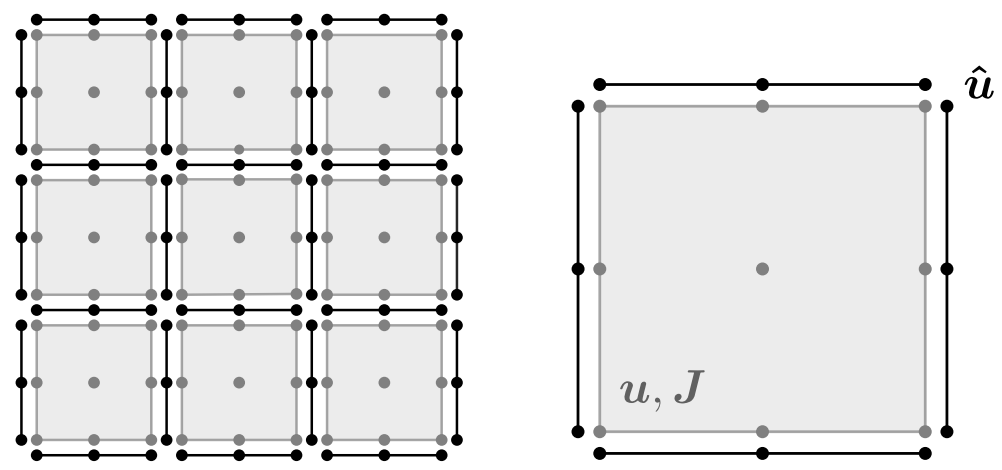

FIGURE 3 Left: HDG discretization of the domain, with the skeleton of the mesh in black. Right: HDG discretization for the local problem in one element. 
where $\mathcal{P}^{p}$ denotes the space of polynomials of degree less or equal to $p$. To keep the notation simple, we use $\boldsymbol{u}, \boldsymbol{J}, \hat{\boldsymbol{u}}$ to denote both the solutions and their approximations.

For an element $K_{i}$, the weak form for the local problem (12) is: given $\hat{\boldsymbol{u}} \in\left[\Lambda^{h}(\Gamma)\right]^{n_{s d}}$, find $\boldsymbol{u} \in\left[\mathcal{P}^{p}\left(K_{i}\right)\right]^{n_{s d}}, \boldsymbol{J} \in\left[\mathcal{P}^{p}\left(K_{i}\right)\right]^{n_{s d} \times n_{s d}}$ such that

$$
\begin{aligned}
& \int_{K_{i}} \boldsymbol{v} \cdot(\boldsymbol{\nabla} \cdot \boldsymbol{\sigma}(\boldsymbol{J}, d)) \mathrm{d} \boldsymbol{V}+\int_{\partial K_{i}} \tau \boldsymbol{v} \cdot((\boldsymbol{C}:[(\hat{\boldsymbol{u}}-\boldsymbol{u}) \otimes \boldsymbol{n}]) \cdot \boldsymbol{n}) \mathrm{d} s=0, \\
& \int_{K_{i}} \boldsymbol{Q}: \boldsymbol{J} \mathrm{d} \boldsymbol{V}+\int_{K_{i}}(\boldsymbol{\nabla} \cdot \boldsymbol{Q}) \cdot \boldsymbol{u} \mathrm{d} V-\int_{\partial K_{i}}(\boldsymbol{Q} \cdot \boldsymbol{n}) \cdot \hat{\boldsymbol{u}} \mathrm{d} s=0,
\end{aligned}
$$

for all $\boldsymbol{v} \in\left[\mathcal{P}^{p}\left(K_{i}\right)\right]^{n_{s d}}$, for all $\boldsymbol{Q} \in\left[\mathcal{P}^{p}\left(K_{i}\right)\right]^{n_{s d} \times n_{s d}}$. Equation $[15 \mathrm{a}$ is derived from (12a) by applying integration by parts, replacing the numerical flux

$$
\hat{\boldsymbol{\sigma}}:=\boldsymbol{\sigma}(\boldsymbol{J}, d)+\tau \boldsymbol{C}:((\hat{\boldsymbol{u}}-\boldsymbol{u}) \otimes \boldsymbol{n})
$$

on the boundary and undoing the integration by parts. The parameter $\tau$ is a positive stabilization parameter, which we will take constant in all the domain. Equation (15b) is obtained by applying integration by parts on (12b) and replacing $\boldsymbol{u}=\hat{\boldsymbol{u}}$ on the element boundary.

The discretization of the local problem (15) leads to a system of the form

$$
\left[\begin{array}{ll}
\boldsymbol{A}_{u u}^{i} & \boldsymbol{A}_{u J}^{i} \\
\boldsymbol{A}_{J u}^{i} & \boldsymbol{A}_{J J}^{i}
\end{array}\right]\left[\begin{array}{c}
\boldsymbol{u}^{i} \\
\boldsymbol{J}^{i}
\end{array}\right]=-\left[\begin{array}{c}
\boldsymbol{A}_{u \hat{u}}^{i} \\
\boldsymbol{A}_{J \hat{u}}^{i}
\end{array}\right] \boldsymbol{\Lambda}^{i},
$$

which can be arranged as the local solver for each element $K_{i}$, expressing $\boldsymbol{u}$ and $\boldsymbol{J}$ in the element in terms of $\hat{\boldsymbol{u}}$,

$$
\boldsymbol{u}^{i}=\mathbf{U}^{K_{i}} \boldsymbol{\Lambda}^{i}, \boldsymbol{J}^{i}=\mathbf{Q}^{K_{i}} \boldsymbol{\Lambda}^{i},
$$

with matrices $\mathbf{U}^{K_{i}}, \mathbf{Q}^{K_{i}}$. The vectors $\boldsymbol{u}^{i}$ and $\boldsymbol{J}^{i}$ are the vectors of nodal values of the element and $\boldsymbol{\Lambda}^{i}$ is the vector of nodal values of $\hat{\boldsymbol{u}}$ on the $n_{f}$ faces of $K_{i}$,

$$
\boldsymbol{\Lambda}^{i}:=\left[\begin{array}{c}
\hat{\boldsymbol{u}}_{i, 1}^{F_{i, 1}} \\
\vdots \\
\hat{\boldsymbol{u}}^{F_{i, n_{f}}}
\end{array}\right] .
$$

For the global problem (13), replacing $\sigma$ by the numerical flux $\hat{\sigma}$, the weak form reads: find $\hat{\boldsymbol{u}} \in\left[\Lambda^{h}(\Gamma)\right]^{n_{s d}}$ such that $\hat{\boldsymbol{u}}=$ $\mathbb{P}_{2}\left(\boldsymbol{u}_{D}\right)$ on $\Gamma_{D}$ and

$$
\int_{\Gamma} \hat{\boldsymbol{v}} \cdot \llbracket \hat{\boldsymbol{\sigma}} \cdot \boldsymbol{n} \rrbracket \mathrm{d} s+\int_{\Gamma_{N}} \hat{\boldsymbol{v}} \cdot(\hat{\boldsymbol{\sigma}} \cdot \boldsymbol{n}) \mathrm{d} s=\int_{\Gamma_{N}} \hat{\boldsymbol{v}} \cdot \boldsymbol{t}_{N} \mathrm{~d} s,
$$

for all $\hat{\boldsymbol{v}} \in\left[\Lambda^{h}(\Gamma)\right]^{n_{s d}}$ such that $\hat{\boldsymbol{v}}=\mathbf{0}$ on $\Gamma_{D}$, where $\mathbb{P}_{2}$ denotes the $L^{2}$ projection onto $\left[\Lambda^{h}(\Gamma)\right]^{n_{s d}}$. Discretizing the global weak form and replacing $\boldsymbol{u}$ and $\boldsymbol{J}$ in terms of $\hat{\boldsymbol{u}}$ by the local solver (18), a system for $\hat{\boldsymbol{u}}$ is obtained. Once $\hat{\boldsymbol{u}}$ is determined, $\boldsymbol{u}$ and $\boldsymbol{J}$ are computed for each element using the local solvers [18).

For this formulation, $\boldsymbol{u}$ converges with order $p+1$ in $L^{2}$ norm and $\boldsymbol{J}$ with order $p+1 / 2$, if an approximation of degree $p$ is used $^{28}$. In a more recent formulation, Sevilla et al ${ }^{26}$ report convergence of order $p+1$ for $\boldsymbol{J}$ based on numerical experiments.

It is important noting that solving the global system of equations only involves the trace variable $\hat{u}$. Thus, the number of degrees of freedom and the computational efficiency are similar to the ones obtained with continuous finite elements with static condensation, see Kirby et al ${ }^{14}$ for a comparison. Nevertheless, HDG provides a suitable framework for very local $h$-refinement without hanging nodes or transition regions of refinement.

\subsection{HDG for the damage equation}

The HDG formulation for the damage field equation (10b), with a given source term $\mathcal{H}^{+}$, can be obtained following the same steps as for the equilibrium equation. Two new variables are defined: $\boldsymbol{q}$ as the gradient of $d$ and $\hat{d}$ as the trace of $d$ on the skeleton of the mesh. The local problems impose the equation in every element $K_{i}$ with Dirichlet boundary conditions, and their weak 
form is: given $\hat{d} \in \Lambda^{h}(\Gamma)$, find $d \in \mathcal{P}^{p}\left(K_{i}\right), q \in\left[\mathcal{P}^{p}\left(K_{i}\right)\right]^{n_{s d}}$ such that

$$
\begin{aligned}
& -\int_{K_{i}} G_{C} l v \boldsymbol{\nabla} \cdot \boldsymbol{q} \mathrm{d} V-\int_{\partial K_{i}} G_{C} l \tau(\hat{d}-d) v \mathrm{~d} s+\int_{K_{i}}\left(\frac{G_{C}}{l}+2 \mathcal{H}^{+}\right) v d \mathrm{~d} V=\int_{K_{i}} v 2 \mathcal{H}^{+} \mathrm{d} V, \\
& \int_{K_{i}} \boldsymbol{w} \cdot \boldsymbol{q} \mathrm{d} V+\int_{K_{i}}(\boldsymbol{\nabla} \cdot \boldsymbol{w}) d \mathrm{~d} V-\int_{\partial K_{i}} \boldsymbol{w} \cdot \boldsymbol{n} \hat{d} \mathrm{~d} s=0,
\end{aligned}
$$

for all $v \in \mathcal{P}^{p}\left(K_{i}\right), \boldsymbol{w} \in\left[\mathcal{P}^{p}\left(K_{i}\right)\right]^{n_{s d}}$. In this case, the numerical flux on the boundary of every element is

$$
\hat{\boldsymbol{q}}:=\boldsymbol{q}+\tau(\hat{d}-d) \boldsymbol{n}
$$

with $\tau$ the stabilization parameter. Also in this case, the stabilization parameter can be any positive value, that here is taken constant in all $\Omega$.

The weak form of the global problem is: find $\hat{d} \in \Lambda^{h}(\Gamma)$ such that

$$
\int_{\Gamma \backslash \partial \Omega} \hat{v} \cdot \llbracket \hat{\boldsymbol{q}} \cdot \boldsymbol{n} \rrbracket \mathrm{d} s=0,
$$

for all $\hat{v} \in \Lambda^{h}(\Gamma)$.

In this case, for degree of approximation $p$, both $d$ and $\boldsymbol{q}$ converge with order $p+1$ in the $L^{2}$ norm. See Cockburn et al ${ }^{27}$ for a proof for the Laplace equation.

Remark 1. Postprocessed damage $d^{*}$. A second element-by-element postprocess can be done to compute a superconvergent solution, $d^{*}$. At every element $K_{i}$, given $d \in \mathcal{P}^{p}\left(K_{i}\right)$ and $q \in\left[\mathcal{P}^{p}\left(K_{i}\right)\right]^{n_{s d}}, d^{*} \in \mathcal{P}^{p+1}\left(K_{i}\right)$ can be computed as the solution of

$$
\begin{aligned}
& \int_{K_{i}} \nabla d^{*} \cdot \nabla v \mathrm{~d} V=\int_{K_{i}} \boldsymbol{q} \cdot \nabla v \mathrm{~d} V \quad \forall v \in \mathcal{P}^{p+1}\left(K_{i}\right), \\
& \int_{K_{i}} d^{*} \mathrm{~d} V=\int_{K_{i}} d \mathrm{~d} V .
\end{aligned}
$$

The postprocessed solution $d^{*}$ converges with order $p+2$ in the $L^{2}$ norm and, since the problem is solved at element level, the computational cost is negligible. See Cockburn et al ${ }^{[29}$ for more details.

\section{3 | Staggered scheme}

We solve the phase-field system of equations 10 by using a staggered scheme to decouple the system. Given the solution at load step $n$, the solution at load step $n+1$ is computed by solving alternately the equilibrium and phase-field equations until convergence is reached, see Algorithm 1.

Remark 2. Evaluation of $\mathcal{H}^{+}$. In order to enable the convergence of the staggered scheme, special care has to be taken in the evaluation of the history field $\mathcal{H}^{+}$. This field is computed using the nodal values of $\boldsymbol{J}$ obtained by solving the equilibrium equation. If $\mathcal{H}^{+}$is evaluated at nodes, it may become negative when interpolated to integration points to solve the damage field equation if we use approximation functions of degree higher than 1. This may lead to unphysical solutions for $d$ and to the non-convergence of the staggered scheme. See ${ }^{30}$ for a numerical example of this behavior. To avoid negative values of $\mathcal{H}^{+}$at integration points, we interpolate $\boldsymbol{J}$ at integration points and then evaluate $\mathcal{H}^{+}$at these points. It is also worth noting that the update of $\mathcal{H}^{+}$in step 2 of Algorithm 1 is done using the value of the field at the previous load step, and not the previous staggered iteration, because the former is a converged solution with physical meaning. This can be seen from the definition of $\mathcal{H}^{+}$in $(9)$.

Remark 3. Postprocessed displacement $\boldsymbol{u}^{*}$. Analogously to the computation of the postprocessed damage $d^{*}$, it is possible to recover a postprocessed displacement $\boldsymbol{u}^{*}$ by solving an element-by-element problem, see Cockburn et al ${ }^{24}$. The postprocessed $\boldsymbol{u}^{*}$ converges with order $p+3 / 2$ in the $L^{2}$ norm when using approximation functions of degree $p$. In this case, since the history field $\mathcal{H}^{+}$is computed using $\boldsymbol{J}$, we are not interested in computing $\boldsymbol{u}^{*}$ at every iteration. Once the staggered scheme has converged for a load step, we can compute $\boldsymbol{u}^{*}$ to have a better approximation for the displacements if desired. 


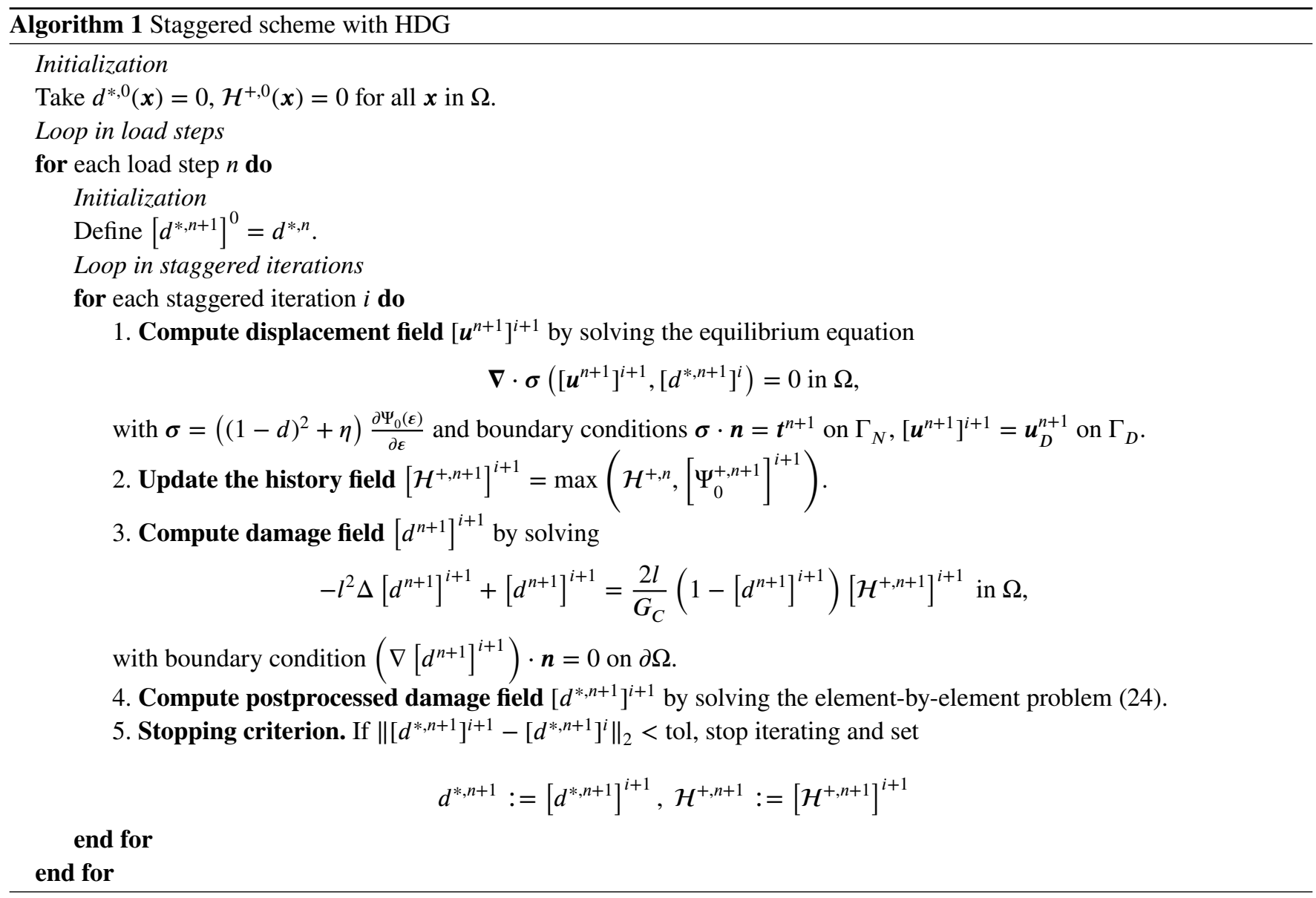

\section{4 | ADAPTIVE REFINEMENT STRATEGY}

The presented HDG formulation for phase-field can be exploited to implement an adaptive refinement strategy which is naturally handled by the method. Because HDG is a Discontinuous Galerkin method, different basis functions can be used to approximate the solution in adjacent elements. In particular, we can $h$-refine the elements along the crack, where more accuracy is needed to capture the solution, with no numerical treatment of the transition between refined and non-refined elements. Here, $p$-refinement is not considered because using significantly high degrees to approximate solutions with steep gradients may give oscillations. However, the ideas are extendable to $p$ and $h p$-refinement.

We consider two types of element, standard and refined, and use different approximation spaces in each one of them. Here, no recursive refinements are contemplated, since the final element size required to capture the solution inside refined elements is determined by the length-scale parameter $l$ in the phase-field model.

In standard elements, the approximation space is the standard one in FEM, this is, $\mathcal{P}^{p}$. In refined elements the space is $h$ refined: given a refinement factor $m$, we consider a uniform submesh of $m \times m$ subelements and we define the approximation space as the sum of the standard approximation spaces for all subelements. For an element $K_{i}$ that is refined, its subelements are denoted as $K_{i, j}$ for $j=1 \ldots m^{2}$. The faces $\Gamma_{f}$ shared by two refined elements, as well as the boundary faces of refined elements, are also refined, meaning that they are divided in subfaces $\Gamma_{f, j}$ for $j=1 \ldots m$. Faces belonging to standard elements are not refined, even the faces shared with a refined element, since we assume the face is far enough from the critical region near the crack, see Figure 4

Thus, the considered approximation spaces for the components of the primal variables $\boldsymbol{u}, \boldsymbol{J}, \boldsymbol{d}$ and the traces $\hat{\boldsymbol{u}}, \hat{\boldsymbol{d}}$ are defined as

$$
\begin{aligned}
& \mathcal{V}_{\text {ref }}^{h}(\Omega)=\left\{v \in L^{2}(\Omega):\left.v\right|_{K_{i}} \in \mathcal{P}^{p}\left(K_{i}\right) \text { for } i \in E_{\text {std }},\left.v\right|_{K_{i}} \in \mathcal{P}_{\text {ref }}^{p}\left(K_{i}\right) \text { for } i \in E_{\text {ref }}\right\}, \\
& \Lambda_{\text {ref }}^{h}(\Gamma)=\left\{\hat{v} \in L^{2}(\Gamma):\left.\hat{v}\right|_{\Gamma_{f}} \in \mathcal{P}^{p}\left(\Gamma_{f}\right) \text { for } f \in F_{\text {std }},\left.\hat{v}\right|_{\Gamma_{f}} \in \mathcal{P}_{\text {ref }}^{p}\left(\Gamma_{f}\right) \text { for } f \in F_{\text {ref }}\right\},
\end{aligned}
$$


where $E_{\text {std }}$ and $E_{\text {ref }}$ are the sets of standard and refined elements, $F_{\text {std }}$ and $F_{\text {ref }}$ are the sets of standard and refined faces, and the refined polynomial spaces are

$$
\begin{aligned}
& \mathcal{P}_{\text {ref }}^{p}\left(K_{i}\right)=\left\{v \in L^{2}\left(K_{i}\right): v \in \mathcal{P}^{p}\left(K_{i, j}\right) \text { for } j=1 \ldots m^{2}\right\}, \\
& \mathcal{P}_{\text {ref }}^{p}\left(\Gamma_{f}\right)=\left\{\hat{v} \in L^{2}\left(\Gamma_{f}\right): \hat{v} \in \mathcal{P}^{p}\left(\Gamma_{f, j}\right) \text { for } j=1 \ldots m\right\} .
\end{aligned}
$$

From the implementation point of view, we define a reference element for each type of element: standard and refined. Standard elements are mapped to the standard reference element as usual. The elements selected to be refined are mapped to the refined reference element, which is actually the one divided in $m^{2}$ uniform subelements. This strategy extremely reduces the number of cases in the implementation.

Inside refined elements, we impose continuity of the solution between subelements in weak form. Keeping an HDG philosophy, we consider the inner skeleton in the element, namely

$$
\mathcal{I}_{i}=\bigcup_{j=1}^{m^{2}} \partial K_{i, j} \backslash \partial K_{i},
$$

and define $\tilde{\boldsymbol{u}}$ and $\tilde{d}$ as the inner traces of the displacements $\boldsymbol{u}$ and the damage $d$, respectively, inside the element, see Figure 4 The local problem in refined elements is consequently modified using the refined local spaces for volume variables, side trace variables and interior trace variables, and accounting for the weak imposition of continuity between subelements. The local problems for standard elements and the global problems are the same as in standard HDG, accounting for the richer space in refined faces in the global problem.

Notice that our approach is equivalent to non-conformal $h$-refinement in standard HDG. We choose to state the formulation with a refined reference element in order to maintain the same structure of the code and to keep the conditionals in the implementation to the minimum. Also, the resulting global system has less degrees of freedom, since the ones corresponding to inner traces are not incorporated into the system.

Remark 4. Continuous vs discontinuous approximation in refined elements. An alternative option would be to consider a piecewise polynomial continuous approximation in refined elements. However, with a mixed formulation as the ones in the local problems, it would require inner stabilization in the element, depending on a stabilization parameter whose tuning may not be straightforward ${ }^{31}$. The considered discontinuous subelement-by-subelement approximation inside refined elements provides a stable approximation for any positive value of the parameter $\tau$, with additional degrees of freedom only at element-level computations.

Remark 5. One-level vs multi-level adaptive strategies. The proposed methodology is designed to achieve very fine discretizations along the crack with minimum spreading, and to reduce to the minimum the cases in the implementation, while keeping

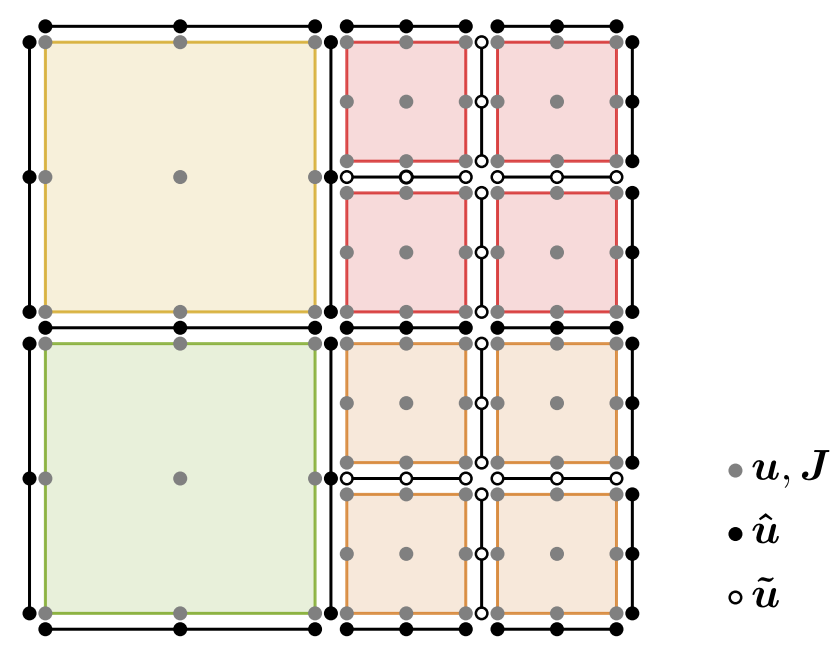

FIGURE 4 Example of an HDG discretization for the equilibrium equation for a mesh of $2 \times 2$ elements with 2 standard elements (left) and 2 refined elements (right). The global problem is solved for the DOFs corresponding only to $\hat{\boldsymbol{u}}$ (black dots). Note that faces shared by a refined and a standard element are not refined. 
the original background mesh. An interesting alternative would be to consider a multi-level refinement strategy ${ }^{6}$, for which an implementation based on finite element libraries for the treatment of hanging nodes would be natural. Nevertheless, we prefer the current proposal for its easier integration in an existing HDG code and, more importantly, because it does not lead to refinement transition zones.

In this section, we first formulate the local problem for refined elements for both the equilibrium and the damage equations. Then, we give some implementation details and show the convergence plots of the formulations for an analytical solution. To simplify the notation, throughout the section we denote as $\hat{K}_{i}$ the union of subelements $K_{i, j}$ and as $\partial \hat{K}_{i}$ the union of exterior faces of subelements.

\section{1 | Local problem for the equilibrium equation for refined elements}

For the equilibrium equation, the strong from for the local problem for a refined element $K_{i}$ is

$$
\begin{cases}\boldsymbol{\nabla} \cdot \boldsymbol{\sigma}(\boldsymbol{J}, d)=\mathbf{0} & \text { in } \hat{K}_{i}, \\ \boldsymbol{J}-\boldsymbol{\nabla} \boldsymbol{u}=\mathbf{0} & \text { in } \hat{K}_{i}, \\ \llbracket \boldsymbol{\sigma} \cdot \boldsymbol{n} \rrbracket=\mathbf{0} & \text { on } \mathcal{I}_{i}, \\ \boldsymbol{u}=\tilde{\boldsymbol{u}}_{i} & \text { on } \mathcal{I}_{i}, \\ \boldsymbol{u}=\hat{\boldsymbol{u}} & \text { on } \partial \hat{K}_{i} .\end{cases}
$$

The new variable $\tilde{\boldsymbol{u}}_{i}$ is the restriction of the displacement field $\boldsymbol{u}$ on the inner skeleton of the element, $\boldsymbol{I}_{i}$. The equilibrium equation on the inner skeleton (28c) has to be incorporated to the system because we are using a discontinuous approximation for the subelements. Continuity is imposed by the condition (28d).

The weak form of the problem reads: given $\hat{\boldsymbol{u}} \in\left[\Lambda_{\mathrm{ref}}^{h}(\Gamma)\right]^{n_{s d}}$, find $\boldsymbol{u} \in\left[\mathcal{P}_{\mathrm{ref}}^{p}\left(K_{i}\right)\right]^{n_{s d}}, \boldsymbol{J} \in\left[\mathcal{P}_{\operatorname{ref}}^{p}\left(K_{i}\right)\right]^{n_{s d} \times n_{s d}}, \tilde{\boldsymbol{u}}_{i} \in\left[\Lambda_{\mathrm{ref}}^{h}\left(\mathcal{I}_{i}\right)\right]^{n_{s d}}$ such that

$$
\begin{aligned}
& \int_{\hat{K}_{i}} \boldsymbol{v} \cdot(\boldsymbol{\nabla} \cdot \boldsymbol{\sigma}(\boldsymbol{J}, d)) \mathrm{d} V+\int_{\partial \hat{K}_{i}} \tau \boldsymbol{v} \cdot((\boldsymbol{C}:[(\hat{\boldsymbol{u}}-\boldsymbol{u}) \otimes \boldsymbol{n}]) \cdot \boldsymbol{n}) \mathrm{d} s+\int_{\mathcal{I}_{i}} 2 \tau \boldsymbol{v} \cdot\left(\left(\boldsymbol{C}:\left[\left(\tilde{\boldsymbol{u}}_{i}-\{\boldsymbol{u}\}\right) \otimes \boldsymbol{n}\right]\right) \cdot \boldsymbol{n}\right) \mathrm{d} s=0, \\
& \int_{\hat{K}_{i}} \boldsymbol{Q}: \boldsymbol{J} \mathrm{d} V+\int_{\hat{K}_{i}}(\boldsymbol{\nabla} \cdot \boldsymbol{Q}) \cdot \boldsymbol{u} \mathrm{d} V-\int_{\partial \hat{K}_{i}}(\boldsymbol{Q} \cdot \boldsymbol{n}) \cdot \hat{\boldsymbol{u}} \mathrm{d} s-\int_{I_{i}} \llbracket \boldsymbol{Q} \cdot \boldsymbol{n} \rrbracket \cdot \tilde{\boldsymbol{u}}_{i} \mathrm{~d} s=0, \\
& \int_{\mathcal{I}_{i}} \tilde{\boldsymbol{v}} \cdot \llbracket \hat{\boldsymbol{\sigma}} \cdot \boldsymbol{n} \rrbracket \mathrm{d} s=0,
\end{aligned}
$$

for all $\boldsymbol{v} \in\left[\mathcal{P}_{\text {ref }}^{p}\left(K_{i}\right)\right]^{n_{s d}}$, for all $\boldsymbol{Q} \in\left[\mathcal{P}_{\text {ref }}^{P}\left(K_{i}\right)\right]^{n_{s d} \times n_{s d}}$, for all $\tilde{\boldsymbol{v}} \in\left[\Lambda_{\text {ref }}^{h}\left(\mathcal{I}_{i}\right)\right]^{n_{s d}}$. The mean operator is defined as $\{\odot\}=$ $\frac{1}{2}\left(\odot_{L_{f}}+\odot_{R_{f}}\right)$, with $L_{f}$ and $R_{f}$ the left and right subelements sharing the face and $\odot_{j}$ the value of $\odot$ from subelement $K_{i, j}$.

The weak form is obtained substituting the numerical flux $\hat{\sigma}$ defined in $(16)$ in the integrals over $\partial K_{i}$ and $\mathcal{I}_{i}$. In this case, the discretization of the weak from leads to a system of the form

$$
\left[\begin{array}{ccc}
\boldsymbol{A}_{u u}^{i} & \boldsymbol{A}_{u J}^{i} & \boldsymbol{A}_{u \tilde{u}}^{i} \\
\boldsymbol{A}_{J_{u}^{i}}^{i} & \boldsymbol{A}_{J J}^{i} & \boldsymbol{A}_{J \tilde{u}}^{i} \\
\boldsymbol{A}_{\tilde{u} u}^{i} & \boldsymbol{A}_{\tilde{u} J}^{i} & \boldsymbol{A}_{\tilde{u} \tilde{u}}^{i}
\end{array}\right]\left[\begin{array}{c}
\boldsymbol{u}_{i} \\
\boldsymbol{J}_{i} \\
\tilde{\boldsymbol{u}}_{i}
\end{array}\right]=-\left[\begin{array}{c}
\boldsymbol{A}_{u \hat{u}}^{i} \\
\boldsymbol{A}_{J \hat{u}}^{i} \\
\mathbf{0}
\end{array}\right] \boldsymbol{\Lambda}_{i},
$$

which gives a local solver for $\boldsymbol{u}$ and $\boldsymbol{J}$ in the element $K_{i}$ with the same structure as (18). One can also obtain the local solver for the elemental variable $\tilde{\boldsymbol{u}}_{i}$. However, it is not used since it is an auxiliary variable in the local problem, not appearing in the global equations.

Eliminating the degrees of freedom corresponding to $\tilde{\boldsymbol{u}}_{i}$ from the global system clearly decreases the size of the matrix. However, the coupling between the remaining degrees of freedom increases, i.e., there are more non-zero entries per row. In fact, the resulting matrix has the same sparsity pattern as for a $p$-refinement. If compared to a standard element, the sparsity pattern is the same but with $m$ times more degrees of freedom in each refined face. See an example in Section 4.5 


\subsection{Local problem for the damage equation for refined elements}

Defining $\tilde{d}_{i}$ as the inner trace of the damage $d$ on $\mathcal{I}_{i}$, the strong form for the local problem for refined elements is

$$
\begin{cases}\left(\frac{G_{C}}{l}+2 \mathcal{H}\right)-G_{C} l \nabla \cdot \boldsymbol{q}=2 \mathcal{H} & \text { in } \hat{K}_{i}, \\ \boldsymbol{q}-\boldsymbol{\nabla} d=\mathbf{0} & \text { in } \hat{K}_{i}, \\ \llbracket \boldsymbol{q} \cdot \boldsymbol{n} \rrbracket=\mathbf{0} & \text { on } \mathcal{I}_{i}, \\ d=\tilde{d}_{i} & \text { on } \mathcal{I}_{i}, \\ d=\hat{d} & \text { on } \partial \hat{K}_{i} .\end{cases}
$$

The corresponding weak form is: given $\hat{d} \in \Lambda_{r e f}^{h}(\Gamma)$, find $d \in \mathcal{P}_{\text {ref }}^{p}\left(K_{i}\right), \boldsymbol{q} \in\left[\mathcal{P}_{\text {ref }}^{p}\left(K_{i}\right)\right]^{n_{s d}}, \tilde{d}_{i} \in \Lambda_{\text {ref }}^{h}\left(\mathcal{I}_{i}\right)$ such that

$$
\begin{aligned}
& -\int_{\hat{K}_{i}} G_{C} l v \boldsymbol{\nabla} \cdot \boldsymbol{q} \mathrm{d} V-\int_{\partial \hat{K}_{i}} G_{C} l \tau(\hat{d}-d) v \mathrm{~d} s-\int_{\mathcal{I}_{i}} 2 G_{C} l \tau\left(\tilde{d}_{i}-\{d\}\right) v \mathrm{~d} s+\int_{\hat{K}_{i}}\left(\frac{G_{C}}{l}+2 \mathcal{H}\right) v d \mathrm{~d} V=\int_{\hat{K}_{i}} v 2 \mathcal{H} \mathrm{d} V, \\
& \int_{\hat{K}_{i}} \boldsymbol{w} \cdot \boldsymbol{q} \mathrm{d} V+\int_{\hat{K}_{i}}(\boldsymbol{\nabla} \cdot \boldsymbol{w}) d \mathrm{~d} V-\int_{\partial \hat{K}_{i}} \boldsymbol{w} \cdot \boldsymbol{n} \hat{d} \mathrm{~d} s-\int_{\mathcal{I}_{i}} \llbracket \boldsymbol{w} \cdot \boldsymbol{n} \rrbracket \tilde{d}_{i} \mathrm{~d} s=0, \\
& \int_{\mathcal{I}_{i}} \tilde{v} \llbracket \hat{\boldsymbol{q}} \cdot \boldsymbol{n} \rrbracket \mathrm{d} s=0,
\end{aligned}
$$

for all $v \in \mathcal{P}_{\text {ref }}^{p}\left(K_{i}\right)$, for all $\boldsymbol{w} \in\left[\mathcal{P}_{\text {ref }}^{p}\left(K_{i}\right)\right]^{n_{s d}}$, for all $\tilde{v} \in \Lambda_{\text {ref }}^{h}\left(\mathcal{I}_{i}\right)$. The numerical flux prescribed on $\partial K_{i}$ and $\mathcal{I}_{i}$ is the same as for the standard elements (22). The structure for the local solver is also preserved in this case.

Remark 6. Postprocessed $d^{*}$ in refined elements. For the postprocess problem to determine the superconvergent solution $d^{*}$ in refined elements, the computations are done at subelement level. No condition on the inner trace $\mathcal{I}_{i}$ needs to be added at the formulation, since the problem is solved at each subelement independently.

\section{3 | Refining criterion}

The phase-field solution needs more resolution along cracks, where it presents sharp variations. The damage field takes values close to 1 near the crack and values close to 0 far from the crack, thus we can use the value of the damage field as an indicator of whether an element needs to be refined or not. In particular, we use the value of the postprocessed damage field $d^{*}$ on the nodes of the element to define the refining criterion. An element $K_{i}$ is refined if a threshold value $d_{\text {ref }}^{*}$ is reached, that is,

$$
i \in E_{\text {ref }} \text { if } \max _{x \in K_{i}} d^{*}(x, t) \geq d_{\text {ref }}^{*} .
$$

Numerically, we have found that a reasonable value for $d_{\text {ref }}^{*}$ is between 0.1 and 0.2 . This will be discussed later in the numerical examples.

The refining criterion is applied at the end of every staggered iteration (in step 4 in Algorithm 17 because, since we are modeling brittle fracture, the crack can grow significantly at a single load step.

Here, coarsening of the discretization in the wake of the crack tip is not considered. As the phase-field solution has sharp variations across the whole crack, resolution is needed not only near the crack tip, but also in the rest of the crack to properly describe its geometry. To coarsen the discretization, one could introduce a sharp crack via X-FEM ${ }^{32}$, following the ideas of continuous-discontinuous transitions in fracture models ${ }^{3334}$, and maintain the refinement only in the vicinity of the crack tip. This idea has been exploited by Patil et al ${ }^{7}$ within a CG setting.

\section{4 | Refined elements and faces}

To integrate over the refined elements and faces, we use two reference elements with the standard and refined approximation spaces, respectively. In this way, we incorporate all the information of the refinement into the reference element, and most computations and assembly of elemental matrices can be done with the standard element routines. We can use a unique refined 

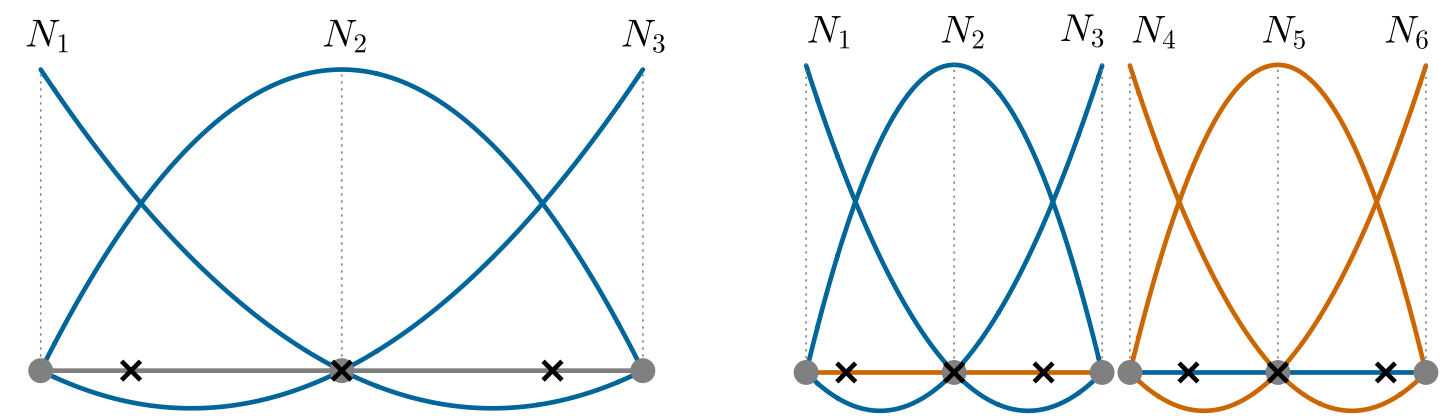

FIGURE 5 Scheme of the discretizations for the standard reference element (left) and for the refined reference element with refinement factor 2 (right) for degree of approximation $p=2$ in 1D. The nodes of the element are represented by grey dots and the integration points by black crosses. The refined element has 6 elemental basis functions.

reference element because the refinement factor is the same in all refined elements, and it can be computed only once in the preprocess. An alternative is to loop over all subelements of refined elements using the standard reference element.

The refined reference element has all the integration points, nodes and shape functions of the HDG discretization of the submesh. The shape functions associated to a subelement are extended with value 0 to the rest of subelements. The refined reference element also includes inner geometrical information for the computation of integrals and jumps on inner faces and the assembly to subelements nodal values.

To illustrate the discretization with subelements, Figure 5 shows a representation of the nodal basis functions and integration points for a 1D standard reference element (on the left) and for a refined reference element (on the right). In this example, the refined element is split in 2 subelements, so it has twice as many shape functions and integration points as the standard element. Analogously, the 2D refined elements in Figure 4 which are split in 4 subelements, have 4 times the number of shape functions and integration points of the standard element. Shared faces between refined elements and boundary faces are also refined with refined discretization as the one examplified in Figure 5

A refined element can have some refined faces and some standard faces. To avoid dealing with different cases on elemental computations, all faces are integrated as if they were refined faces. Then, before assembly, a projection operator is applied for standard faces, using the fact that the standard space is included in the refined one.

\section{5 | Convergence study}

To test the proposed formulation and its implementation, we study the convergence of the numerical solution to a known analytical solution. Since the problem in refined elements is formulated to be equivalent to non-conformal $h$-refinement with HDG, the expected orders of convergence are the same that are proved for the standard HDG formulations. For a fixed computational mesh, we study the convergence when refining all of its elements for an increasing refinement factor $m$.

Consider a square domain $\Omega=[0,1]^{2}$ with a computational mesh of $10 \times 10$ elements. For the equilibrium equation, we set the body force and Dirichlet boundary conditions on $\partial \Omega$ corresponding to the analytical solution

$$
\boldsymbol{u}(x, y)=\left(\begin{array}{c}
\exp (0.1 \sin (5.1 x+6.2 y)) \\
\exp (0.3 \cos (4.3 x+30.4 y))
\end{array}\right)
$$

considering a given damage field $d(x, y)=(\sin (x+y)+1) / 5$, with material parameters $E=20 \mathrm{GPa}, \nu=0.3$ and numerical parameter $\tau=100$. For refinement factors $m=1,2,4,8$, the convergence plots obtained are shown in Figure 6 with a slightly better convergence than the expected one: orders of convergence in the $L^{2}$ norm are $p+1$ and $p+1 / 2$ for the displacement field $\boldsymbol{u}$ and its gradient $\boldsymbol{J}$, respectively, if degree of approximation $p$ is used.

For the damage equation, we impose Dirichlet boundary conditions on $\partial \Omega$ and the source function $\mathcal{H}$ corresponding to the solution

$$
d(x, y)=\frac{\sin (3 x+y)+1}{3},
$$

with parameters $G_{C}=8.9 \cdot 10^{-5} \mathrm{kN} / \mathrm{mm}, l=0.01 \mathrm{~mm}, \tau=100$. In this case, the expected orders of convergence are $p+1$ for the damage field $d$ and $p+2$ for the postprocessed damage field $d^{*}$. Figure 7 shows the convergence plots obtained for this equation 
on the same $10 \times 10$ mesh and for refinement factors $m=2,4,8,16$, again in agreement with the theoretical convergence rates for uniform $h$-refinement.

Whether to condensate or not the interior traces in refined elements is an implementation decision that does not affect the numerical solution, but it has an important effect in the resulting global system of equations. As an example, a mesh with $10 \times 10$ elements with refinement factor $m=8$ and a mesh with $10 m \times 10 m$ elements without refinement are considered. These discretizations are equivalent in the sense that they lead to the same numerical solution, but the degrees of freedom corresponding to inner traces are not incorporated into the global system in the discretization with refinement. Figure 8 shows the sparsity patterns for degree $p=2$. The matrix for $10 \mathrm{~m} \times 10 \mathrm{~m}$ standard elements (left plot) has size 75840 , and the number of non-zero coefficients per row is 42 , when not affected by the boundary. For the matrix corresponding to $10 \times 10$ refined elements (right plot) the number of non-zero coefficients per row increases to 336, due to the fact that now each refined face has 8 times more degrees of freedom than a standard face, but the size of the matrix is significantly reduced to 8640 . That is, the condensation of the interior traces leads to more coupling of degrees of freedom, but also to a reduced dimension and number of non-zero entries in the resulting global matrix.

In Figure 9, we compare the CPU time needed for the direct solver ( $\backslash$ operator in Matlab) to solve the two systems, for different values of the refinement level $m$ and degree $p$. The ratio of CPU times between the HDG discretization with refinement and the corresponding standard discretization is below 1 in all cases. That is, condensating the interior trace leads to a saving in CPU time for the direct linear solver.

\section{5 | NUMERICAL EXPERIMENTS}

In this section, we test the ability of the proposed adaptive strategy to model propagating cracks in four different examples: three well-known benchmark tests in fracture simulation and a new test with crack branching in the quasi-static regime, with no need of material heterogeneities to trigger the bifurcation.

In the equilibrium equation, plane strain conditions are assumed. In all examples, we iterate over the staggered scheme until convergence is reached with a tolerance of $10^{-2}$ for the postprocessed damage field $d^{*}$. The HDG stabilization parameter, for both the equilibrium and the damage equations, is taken as $\tau=100$.
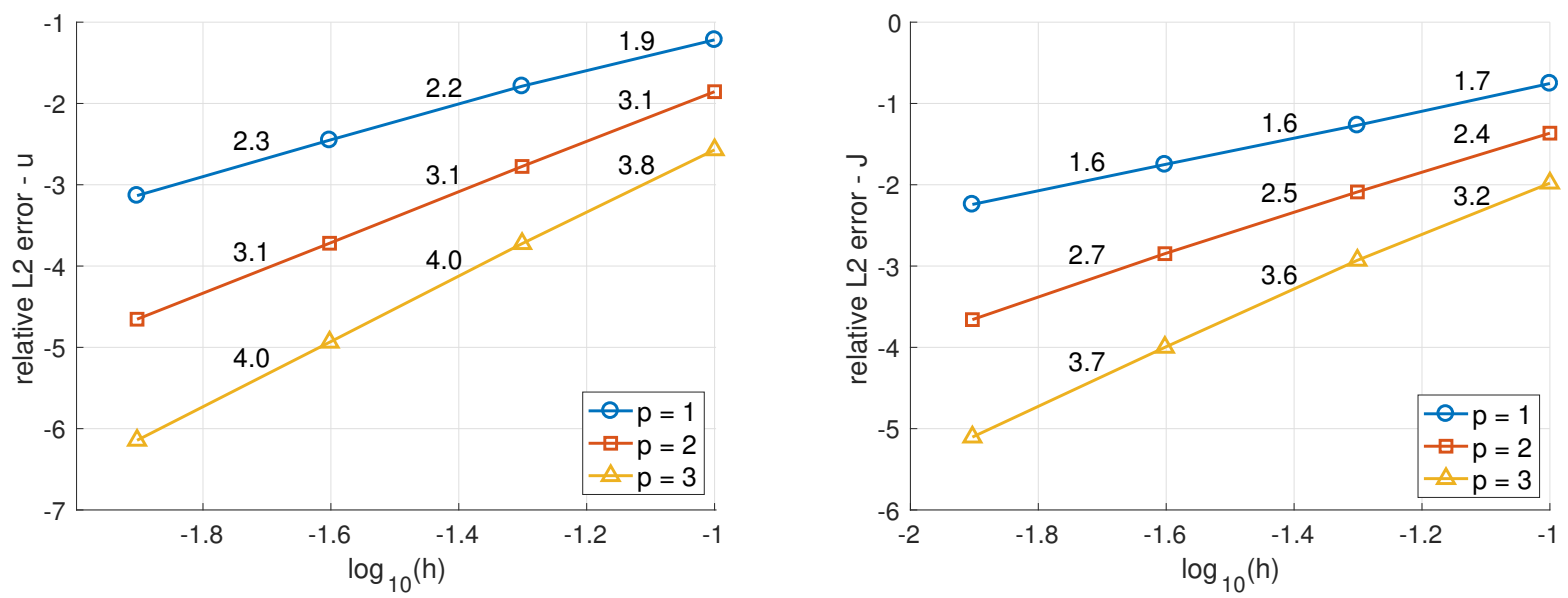

FIGURE 6 Equilibrium equation. Convergence plots obtained for a fixed mesh of $10 \times 10$ elements when refining its elements, for degrees of approximation $p=1,2,3$, for $\boldsymbol{u}$ (left) and $\boldsymbol{J}$ (right). $h$ is the subelement size and the numbers are the slopes in each segment. 

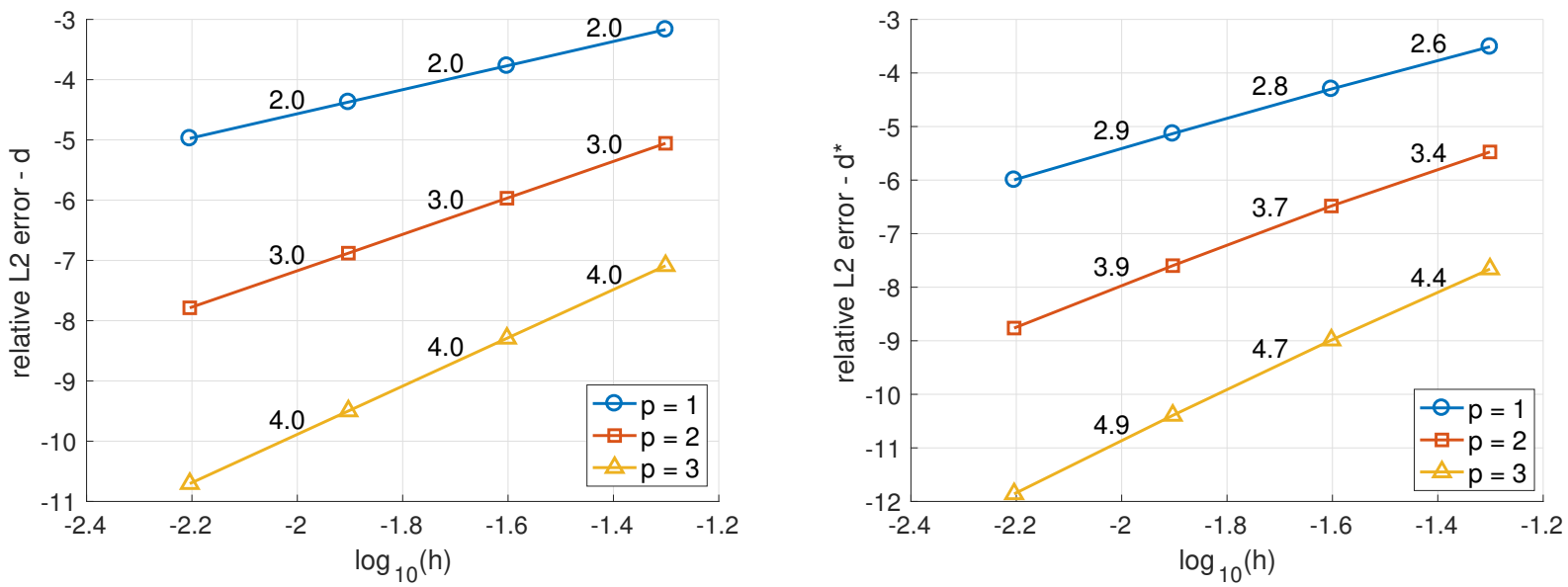

FIGURE 7 Damage equation. Convergence plots obtained for a fixed mesh of $10 \times 10$ elements when refining its elements, for degrees of approximation $p=1,2,3$, for $d$ (left) and $d^{*}$ (right). $h$ is the subelement size and the numbers are the slopes in each segment.
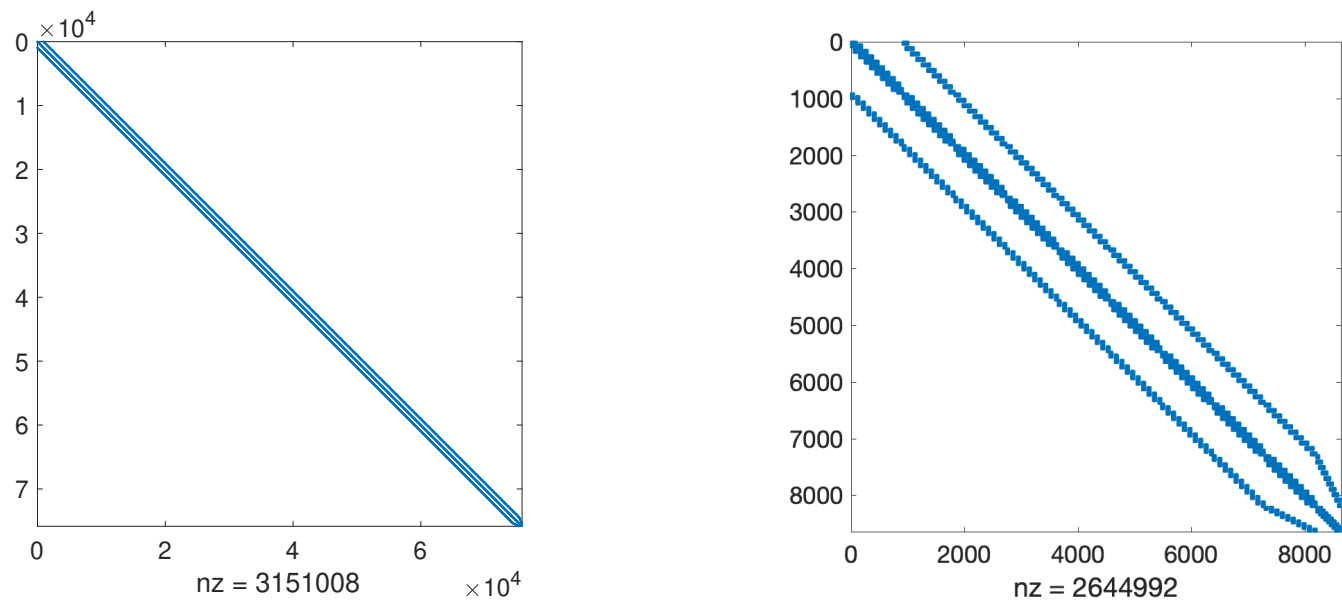

FIGURE 8 Equilibrium equation. Sparsity of matrices: (left) standard HDG with $80 \times 80$ elements and (right) HDG with a $10 \times 10$ elements and refinement factor $m=8$. Degree of approximation $p=2$.

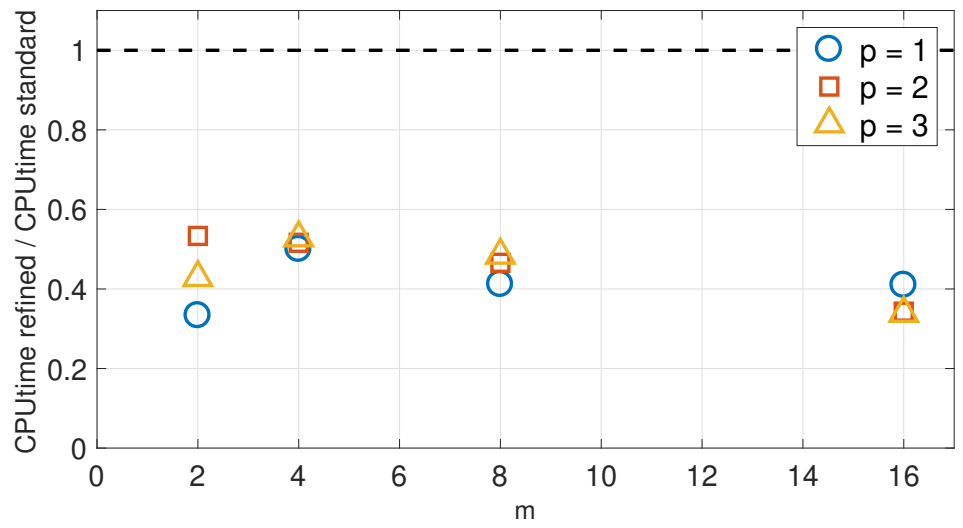

FIGURE 9 Equilibrium equation. Comparison of CPU times for the direct solver. CPU time for the HDG discretization with refinement over the CPU time for standard HDG, for the considered meshes with $10 \mathrm{~m} \times 10 \mathrm{~m}$ elements (equivalently $10 \times 10$ with refinement factor $m$ in all elements) and degrees of approximation $p=1,2,3$. 


\section{1 | Single-edge notched shear test}

With this example, we illustrate the robustness of the proposed adaptive strategy and study the influence of the refinement threshold value $d_{\text {ref }}^{*}$, this is, the value of damage that triggers the refinement. Results obtained with locally and dynamically refined discretizations are compared to the results of a globally refined mesh.

Consider a square plate pre-cracked at mid-height as shown in Figure 10 The plate is fixed on its bottom edge and is subjected to an imposed horizontal displacement on its top edge. Following Ambati et al ${ }^{11}$, the material parameters are $E=210 \mathrm{GPa}$, $v=0.3$ and $G_{C}=2.7 \cdot 10^{-3} \mathrm{kN} / \mathrm{mm}$. The length scale parameter considered is $l=0.015 \mathrm{~mm}$ and the residual stiffness is $\eta=10^{-5}$. The increment of displacement for the load process is $\Delta u_{D}=10^{-4} \mathrm{~mm}$. Computations are done with degree $p=1$, except for the postprocessed damage $d^{*}$ which is approximated with degree $p+1=2$.

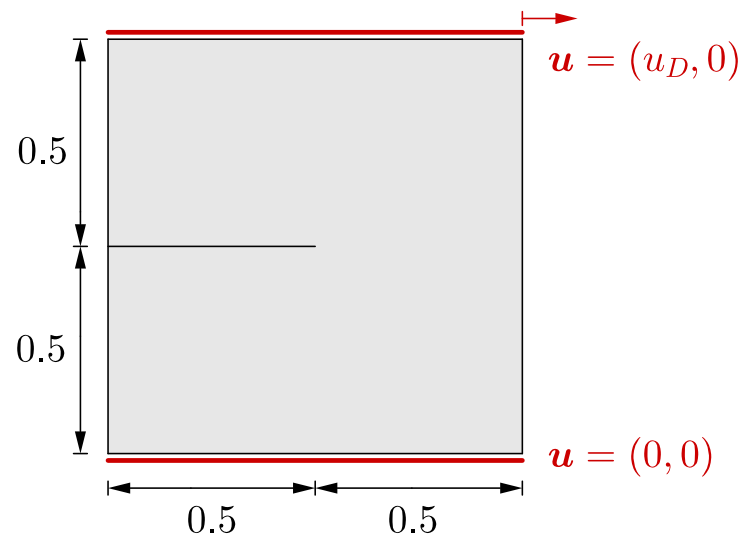

FIGURE 10 Shear test. Geometry and boundary conditions. Dimensions in mm.

As a reference solution, the domain is discretized with a uniform quadrilateral mesh of $240 \times 240$ elements. Three coarser uniform meshes with $48 \times 48,24 \times 24$ and $12 \times 12$ elements are also considered with the corresponding refinement factor $m=5,10,20$ so that the characteristic size of the subelements is $h=1 / 240 \mathrm{~mm}$ in refined elements for all discretizations.

We start by discussing the influence of the refinement value. Figure 11 shows the load-displacement curves obtained for refinement values $d_{\text {ref }}^{*}=0.1$ and $d_{\text {ref }}^{*}=0.2$. Results obtained with the adaptive strategy are very similar to the reference solution. The peaks appearing in the curves corresponding to the coarsest initial mesh of $12 \times 12$ elements coincide with the refinement of elements as the crack propagates, and are interpreted as corrections when spatial resolution is enhanced: the discretization is too coarse in standard elements to properly solve the equilibrium equation with $d$ close to 0 , but the adaptive algorithm still provides reasonable results.

Now we consider a discretization with $24 \times 24$ elements and refinement factor of 10 , with several values $d_{\text {ref }}^{*}=0.1,0.2,0.5$ and 0.7. The load-displacement curves are plotted in Figure 12 If the refinement value is too high, the corrections once elements become refined are not enough to capture the solution and the curve differs significantly from the reference one. A refinement value $d_{\text {ref }}^{*}$ between 0.1 and 0.2 provides good results. Moreover, our experience is that in this range of values the refinement is kept local near cracks.

The reduction of degrees of freedom is substantial when considering the adaptive strategy. Table 1 depicts the number of degrees of freedom for the global problem of the equilibrium equation for each one of the discretizations and for two different refinement values $d_{\text {ref }}^{*}, 0.1$ and 0.2 . Since we use a uniform mesh in all the domain and the refinement is very local near the crack, less degrees of freedom are needed for coarser initial meshes. For the mesh with $24 \times 24$ elements and refinement factor $m=10$, we obtain accurate results with only $1.6-2.1 \%$ of the degrees of freedom of the reference discretization. For the coarsest mesh, with $12 \times 12$ elements and $m=20$, the approximation obtained is reasonable given the limitation of the discretization to resolve the mechanical problem, and with only about $1 \%$ of the number of degrees of freedom.

Figure 13 shows the damage field obtained with these discretizations at three different load steps when a refinement value of $d_{\text {ref }}^{*}=0.2$ is used. The damage path obtained with the considered dynamically changing discretizations is esentially the same as the one of the reference solution. This can be seen more clearly in Figure 14, where the contour plot corresponding to $d^{*}=0.9$ is depicted for the different discretizations, showing an excellent agreement of the crack tip position at the final load step $u_{D}=0.02$ 

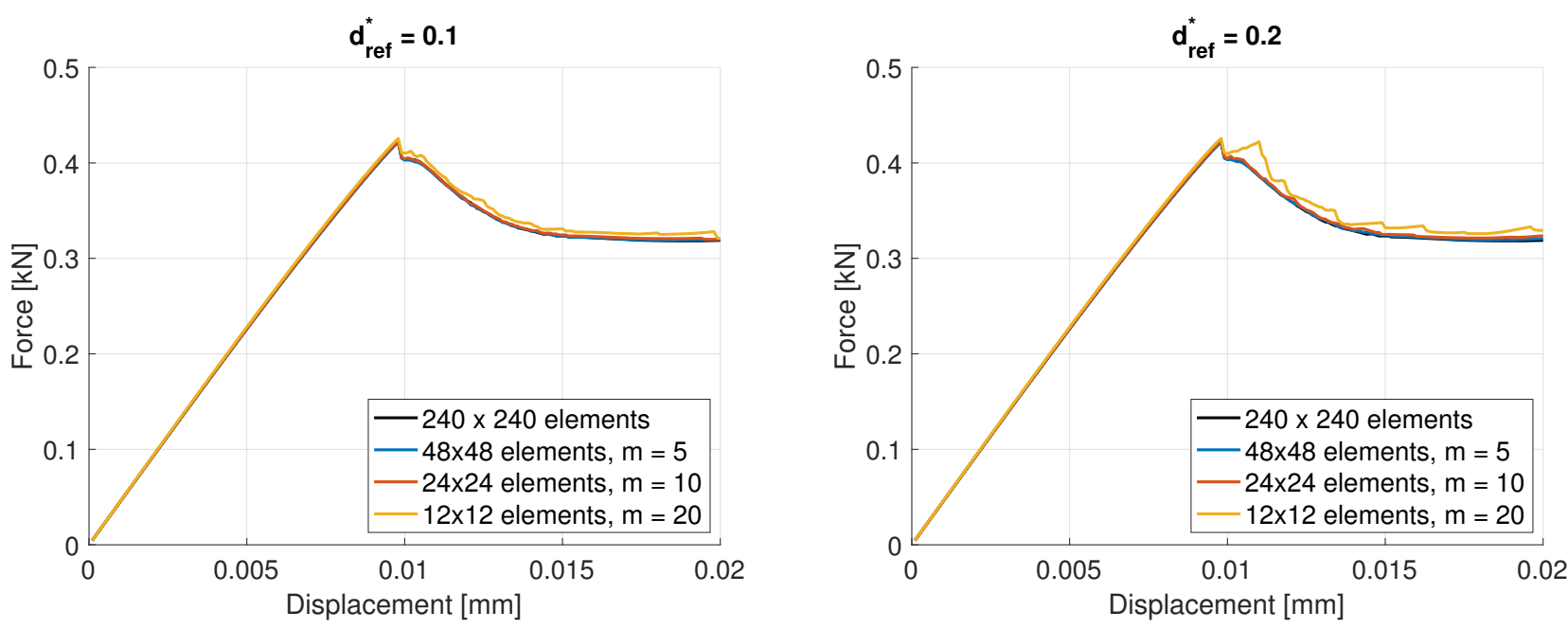

FIGURE 11 Shear test. Load-displacement curves for different discretizations and degree of approximation $p=1$ for refinement values $d_{\text {ref }}^{*}=0.1$ (left) and $d_{\text {ref }}^{*}=0.2$ (right).

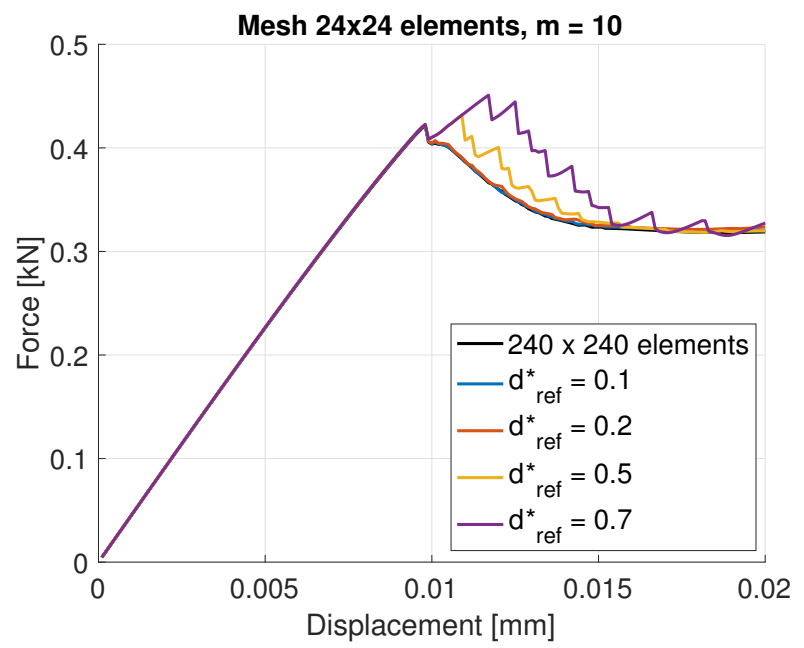

FIGURE 12 Shear test. Load-displacement curves for mesh $24 \times 24$ with refinement $10 \times 10$ and different refinement values. Degree of approximation $p=1$.

$\mathrm{mm}$. We can conclude that refining locally along the crack is enough to capture the solution and that the adaptive strategy is robust with respect to the discretization, as long as elements are refined appropriately to resolve the length scale parameter $l$.

\section{2 | L-shaped panel test}

In this example, we test the performance of the proposed strategy for higher degrees of approximation. Consider an L-shaped plate with geometry and prescribed boundary conditions as shown in Figure 15 The same material parameters as in Ambati et al ${ }^{1}$ are employed, this is, $E=25.8423 \mathrm{GPa}, v=0.18$ and $G_{C}=8.9 \cdot 10^{-5} \mathrm{kN} / \mathrm{mm}$. A length scale parameter $l=2 \mathrm{~mm}$, a residual stiffness $\eta=10^{-5}$ and an increment for the imposed displacements of $\Delta u_{D}=10^{-3} \mathrm{~mm}$ are used. The refinement value is $d_{\mathrm{ref}}^{*}=0.1$.

A uniform quadrilateral mesh with mesh size $h=10 \mathrm{~mm}$ is employed, with three discretizations given by degrees of approximation $p=1, p=2$ and $p=4$. At the beginning, none of the elements are refined. Equivalent discretizations for refined 

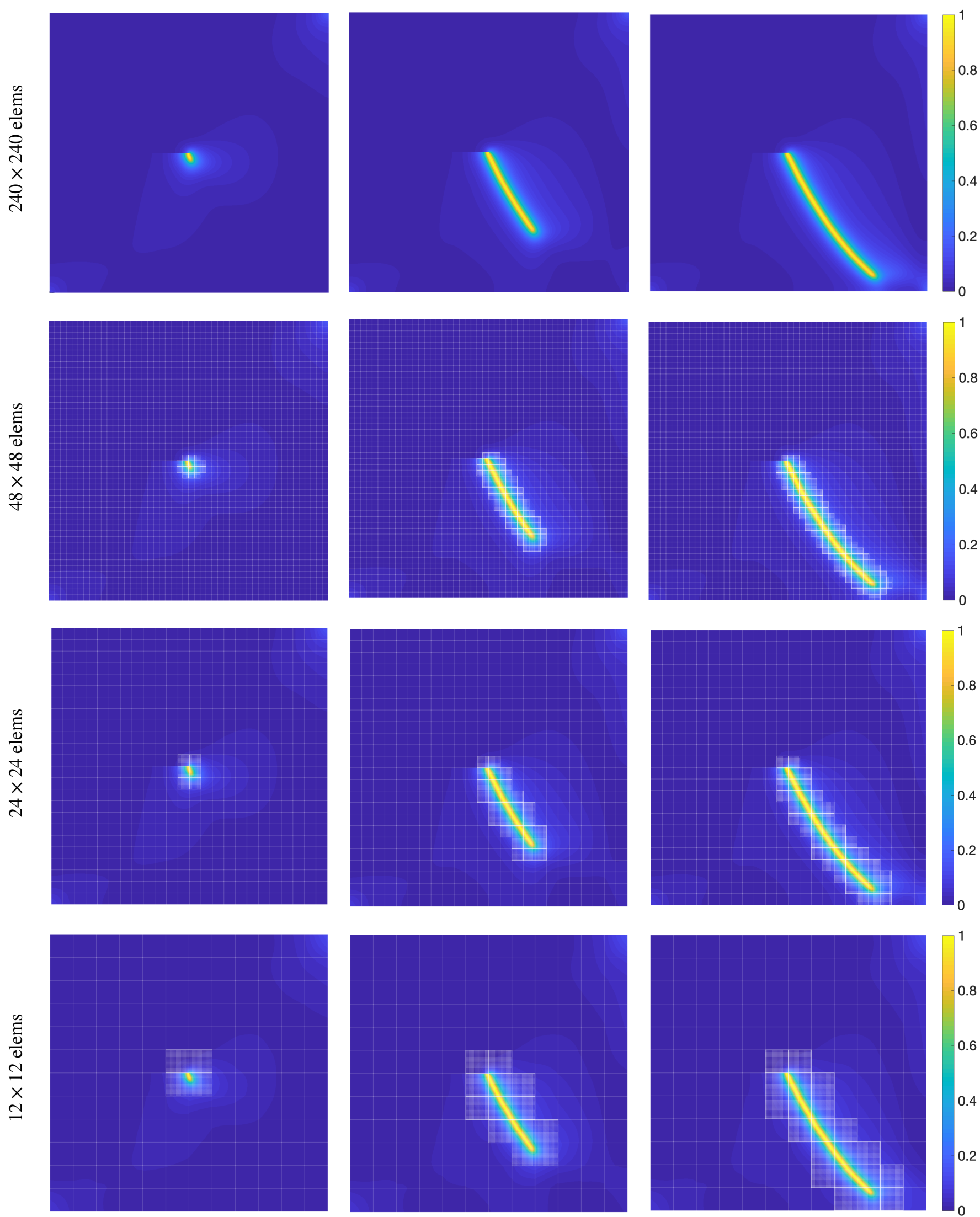

$$
u_{D}=0.010 \mathrm{~mm}
$$

$$
u_{D}=0.014 \mathrm{~mm}
$$

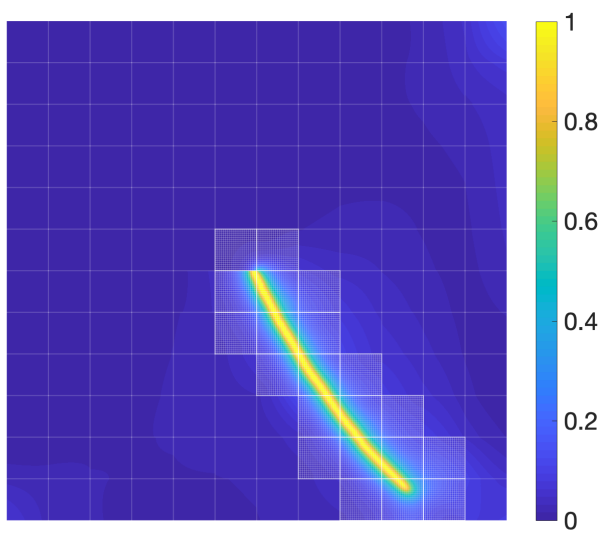

$$
u_{D}=0.020 \mathrm{~mm}
$$

FIGURE 13 Shear test. Damage field for various imposed displacements, with degree of approximation $p=1$ and refinement value $d_{\text {ref }}^{*}=0.2$. 
TABLE 1 Shear test. Comparison of DOFs for different discretizations with linear approximation functions.

\begin{tabular}{lccrrr}
\hline Mesh & Refinement factor & $d_{\text {ref }}^{*}$ & Initial \#DOFs & Final \#DOFs & \%\#DOFs \\
\hline $240 \times 240$ & 0 & - & 461280 & 461280 & $100 \%$ \\
\hline $48 \times 48$ & 5 & 0.1 & 18608 & 25920 & $5.6 \%$ \\
& & 0.2 & 18608 & 22736 & $4.9 \%$ \\
\hline $24 \times 24$ & 10 & 0.1 & 4836 & 9480 & $2.1 \%$ \\
& & 0.2 & 4836 & 7572 & $1.6 \%$ \\
\hline $12 \times 12$ & 20 & 0.1 & 1556 & 4596 & $1.0 \%$ \\
& & 0.2 & 1556 & 3684 & $0.8 \%$ \\
\hline
\end{tabular}
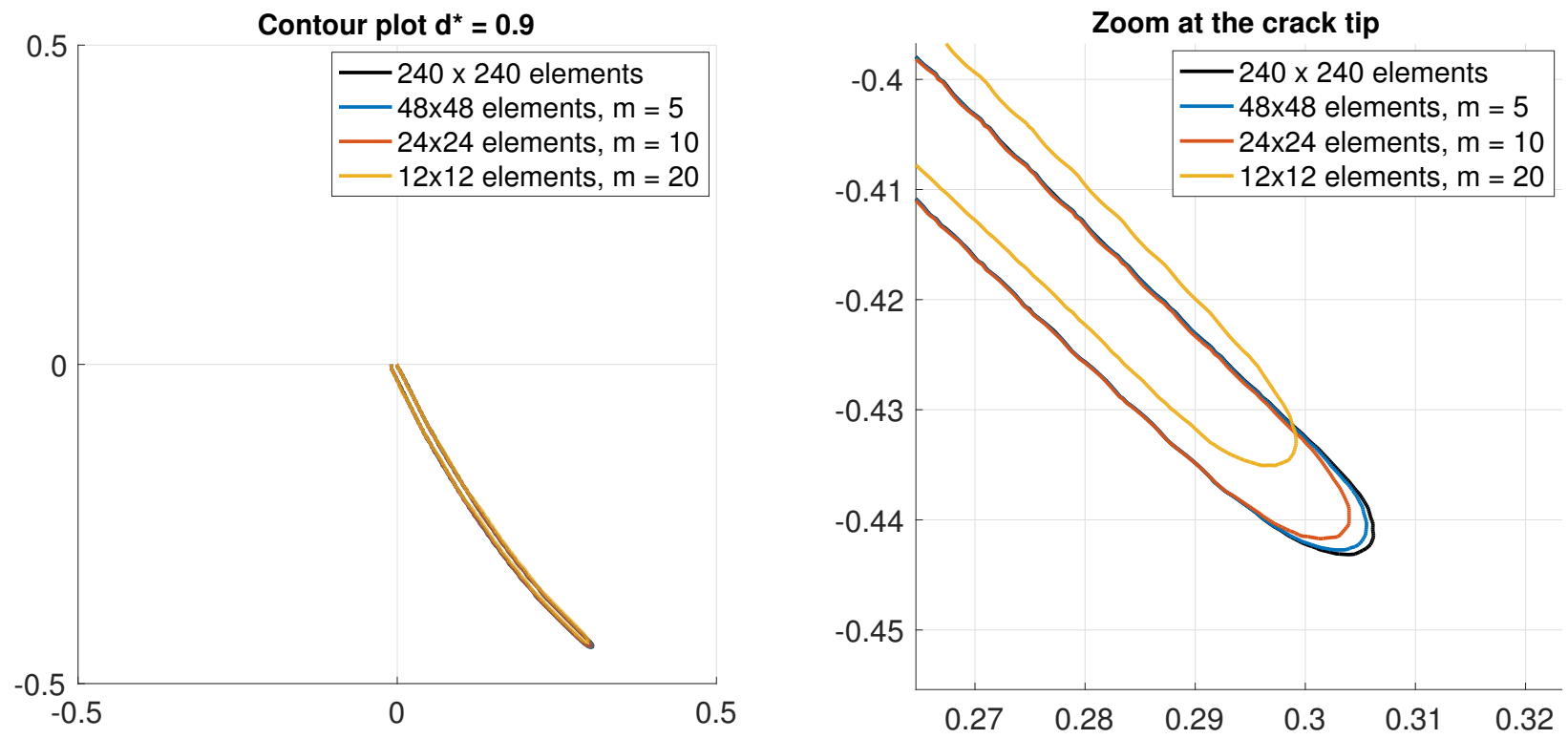

FIGURE 14 Shear test. Left: contour plot at $d^{*}=0.9$ for the different discretizations considered at imposed displacement $u_{D}=0.020 \mathrm{~mm}$, with degree of approximation $p=1$ and refinement value $d_{\mathrm{ref}}^{*}=0.2$. Right: zoom at the crack tip.

elements are used: for the case $p=1$ the refinement factor is $m=20$, for $p=2$ we refine by factor $m=10$ and for $p=4$ we refine by factor $m=5$. Figure 16 shows the crack evolution for the different degrees of approximation and the corresponding load-displacement curves are depicted in Figure 17

The adaptive strategy performs correctly for all cases giving very similar results, showing again the robustness of the proposed strategy and its capability to resolve the crack growth with an automatic adaptation, now with different degrees of approximation. Nonetheless, it is worth mentioning that when using high-order approximations, one must ensure that the refinement is fine enough to properly capture the sharp variation of the phase-field variable in order to avoid oscillations.

\section{3 | Notched plate with a hole}

Since the proposed refinement is done in the reference element, the method also works for non-structured meshes. In this example, we consider a domain with a hole to illustrate the performance of the strategy in this scenario.

This test was first proposed by Ambati et al ${ }^{1}$. Consider a notched specimen with a non-centered hole as shown in Figure 18 The plate is fixed on the lower pin and has imposed vertical displacement on the top pin. The parameters are $E=6 \mathrm{GPa}$, $v=0.22$ and $G_{C}=2.28 \cdot 10^{-3} \mathrm{kN} / \mathrm{mm}$. The length scale parameter is $l=0.5 \mathrm{~mm}$ and the residual stiffness is $\eta=10^{-5}$. We use fixed displacement increments of $\Delta u_{D}=10^{-2} \mathrm{~mm}$. 


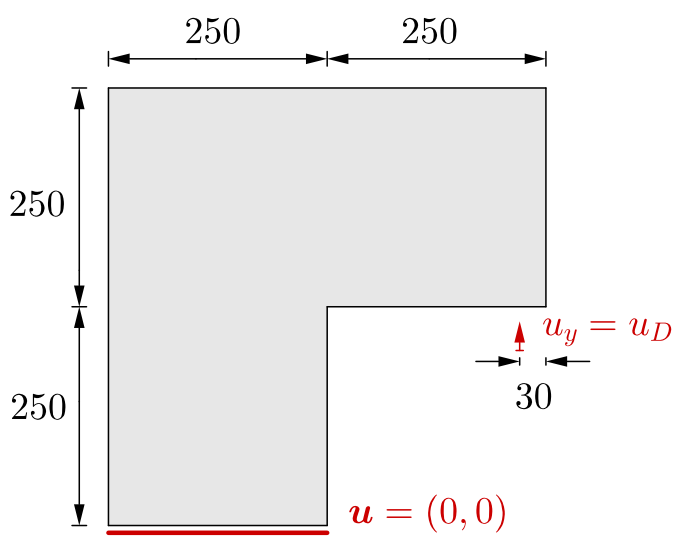

FIGURE $15 L$-shaped test. Geometry and boundary conditions. Dimensions in mm.

We consider a non-structured mesh of quadrilaterals, with element size $h \simeq 5 \mathrm{~mm}$, and degree of approximation $p=2$, see Figure 18 The refinement factor is $m=20$ and we refine using the threshold value $d_{\text {ref }}^{*}=0.2$. The pre-existing crack is defined by a history variable $\mathcal{H}^{+}$as described in Borden et al ${ }^{5}$, refining the elements that contain it.

Figure 19 shows the damage field obtained at different load steps. As expected, the crack is attracted to the hole and a second crack appears in the other side. Elements along the crack are dynamically refined. Elements surrounding the lower pin are also refined because the threshold refinement value is reached in them.

\section{4 | Single-edge notched branching test}

We propose an example of crack branching for quasi-static models of fracture. In contrast with other examples that can be found on the literature, here the branching is caused by the boundary conditions and not by any material heterogeneity. This example illustrates the suitability of the adaptive strategy for complex crack patterns.

Consider a square plate occupying the domain $[-1,1]^{2} \mathrm{~mm}^{2}$, with a pre-crack at mid height of length $0.1 \mathrm{~mm}$, see Figure 20 The plate is clamped on its right edge and vertical displacements are imposed on the top and bottom edges following a parabolic function, this is, $f(x)=u_{D}(x-1)^{2} / 8$. The crack is expected to propagate horizontally up to a certain point and then, because of the clamped right edge, it is expected to branch. The branching point is unknown and, moreover, will strongly depend on the material parameters and profile of prescribed displacements. The proposed adaptive strategy enables to consider a uniform computational mesh in all the domain and the discretization will refine accordingly to the crack evolution.

The material and model parameters used are $E=20 \mathrm{GPa}, v=0.3, G_{C}=8.9 \cdot 10^{-5} \mathrm{kN} / \mathrm{mm}, l=0.0075 \mathrm{~mm}$ and $\eta=10^{-5}$. The loading process is governed by an increment of $\Delta u_{D}=10^{-4} \mathrm{~mm}$. The problem is solved using a uniform quadrilateral mesh of $41 \times 41$ elements with refinement factor $m=15$, linear shape functions and refinement value $d_{\text {ref }}^{*}=0.2$. Again, the initial crack is defined following Borden et a ${ }^{5}$.

Figure 21 shows the damage field obtained at different load steps. The complete evolution of the crack, and of the refinement, can be seen in the YouTube video 13 . The adaptive strategy enables to approximate the branching of the crack automatically, with no need of remeshing.

\section{6 | CONCLUSIONS}

We present an adaptive phase-field model, with very local non-conforming refinement. The key ingredient of our proposal is that it is based on the HDG discretization technique, rather than the standard CG method. For our purposes, the most attractive feature of HDG is that approximation functions are discontinuous across elements, and then continuity of the solution is weakly imposed. This enables the use of different approximation spaces in adjacent elements. In particular, we have considered approximation spaces with different characteristic length $h$. As a result, a coarse mesh can be very locally refined as cracks propagate, with any desired refinement factor $m$ and no special treatment of the transition zone. 

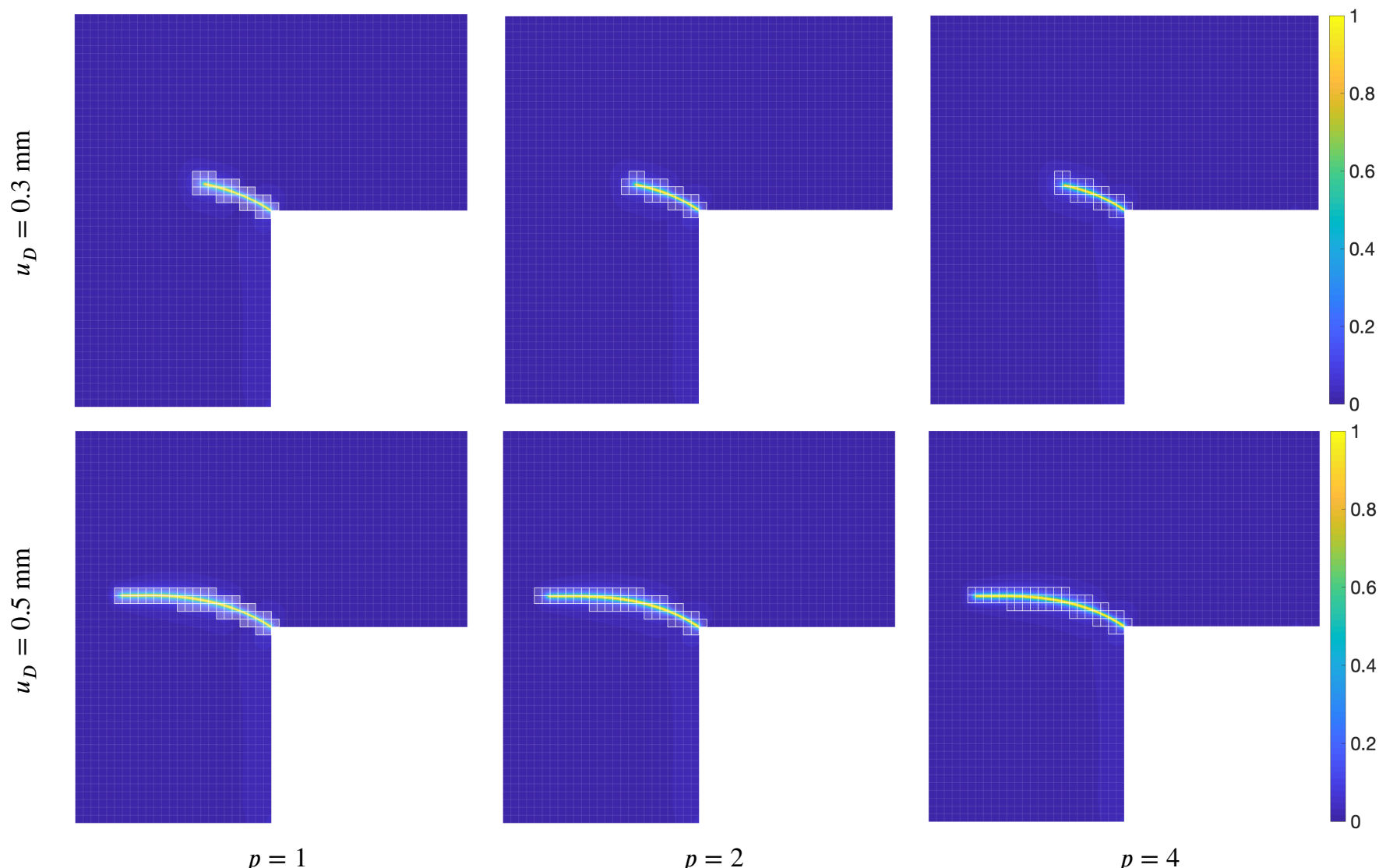

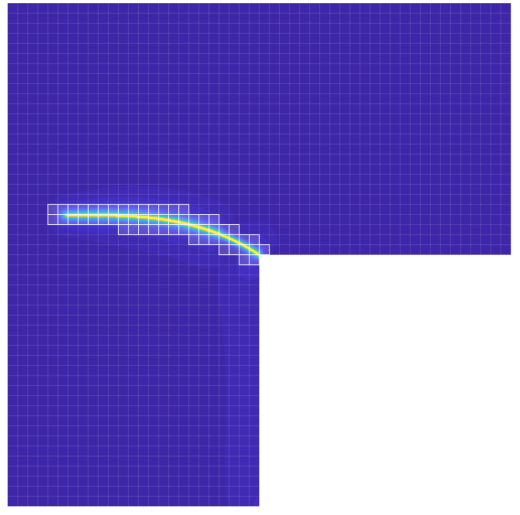

$p=2$

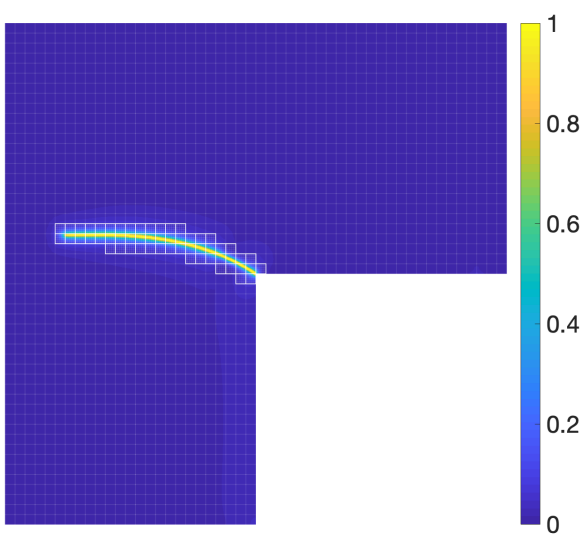

$p=4$

FIGURE $16 L$-shaped test. Damage field at $u_{D}=0.3 \mathrm{~mm}$ and $u_{D}=0.5 \mathrm{~mm}$ obtained with degrees of approximation $p=1,2,4$ and corresponding refinement factors $m=20,10,5$, respectively.

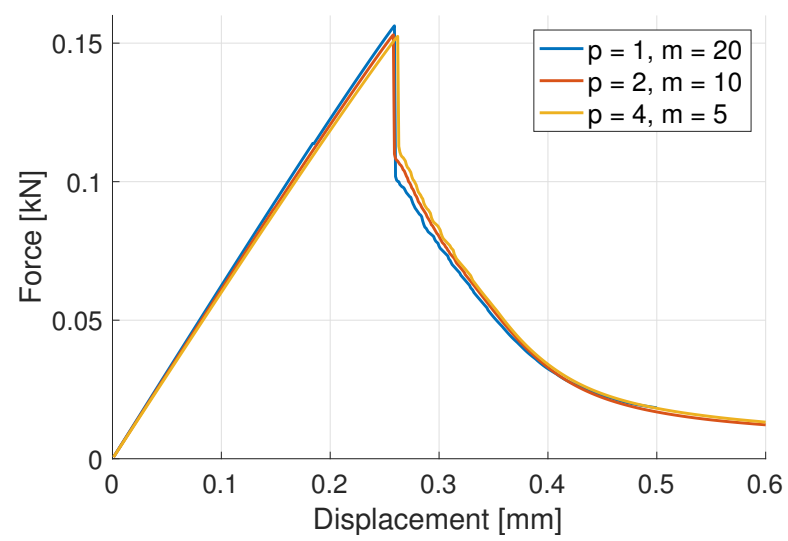

FIGURE 17 L-shaped test. Load-displacement curves for degrees of approximation $p=1, p=2$ and $p=4$ with refinement factor $m$. The threshold refinement value is $d_{\text {ref }}^{*}=0.1$.

An implementation based on a standard and a refined reference element is proposed, keeping the original background mesh fixed during all the simulation and the standard structure of the HDG code.

We illustrate the adaptive strategy in various numerical examples, including a new branching test, with refinement factors up to $m=20$ and degrees up to $p=4$. Numerical results demonstrate the accuracy and robustness of the strategy regarding the crack path, load-displacement curves and position of the crack tip. Also, since the refinement is done at the reference element, the method can be used for non-structured background meshes. 

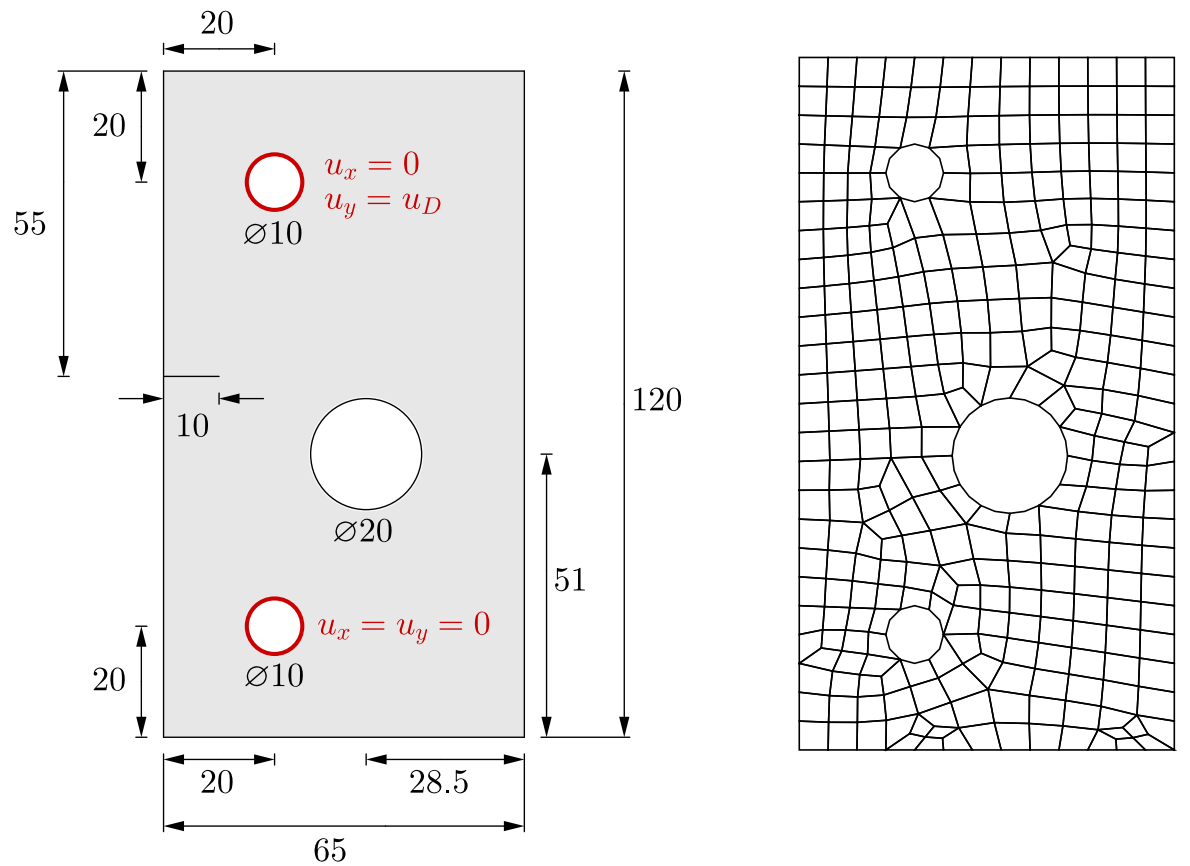

FIGURE 18 Plate with a hole. Left: Geometry and boundary conditions. Dimensions in mm. Right: Computational mesh.

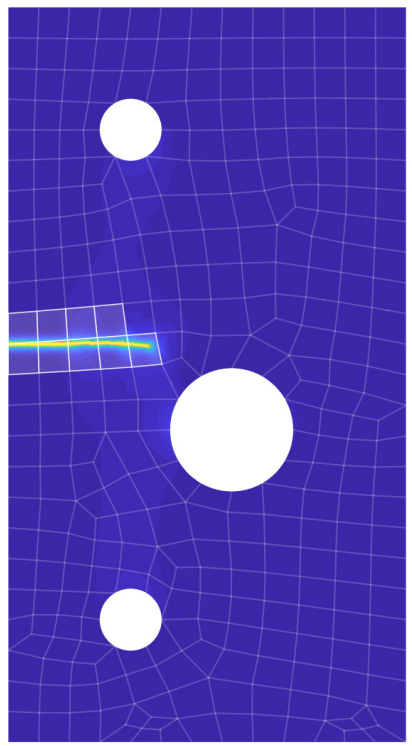

$u_{D}=0.31 \mathrm{~mm}$

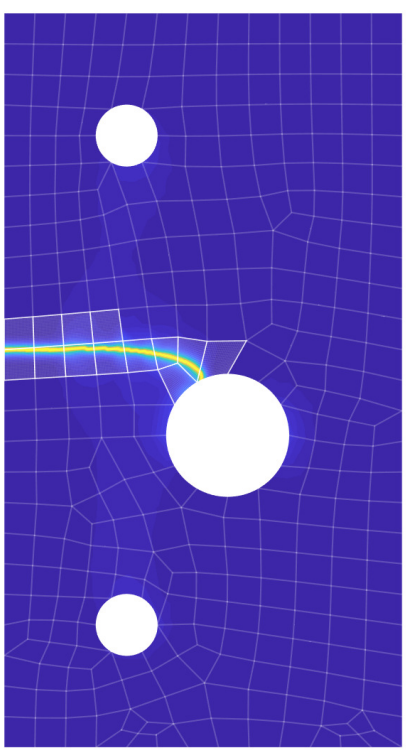

$u_{D}=0.5 \mathrm{~mm}$

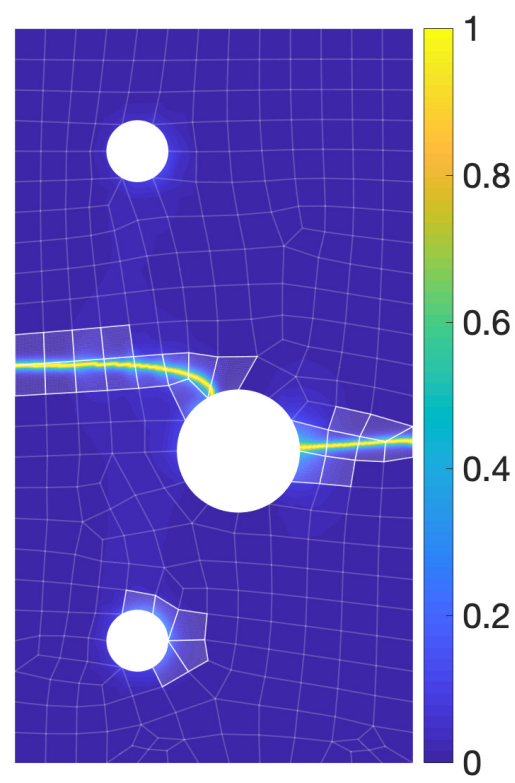

$u_{D}=1.07 \mathrm{~mm}$

FIGURE 19 Plate with a hole. Damage field at various load steps for degree of approximation $p=2$, refinement factor $m=20$ and refinement threshold value $d_{\text {ref }}^{*}=0.2$.

\section{ACKNOWLEDGMENTS}

This work was supported by the AGAUR training grant FI-DGR 2017, the DAFOH2 project (Ministerio de Economía y Competitividad, MTM2013-46313-R) and the Generalitat de Catalunya (2017-SGR-1278). 


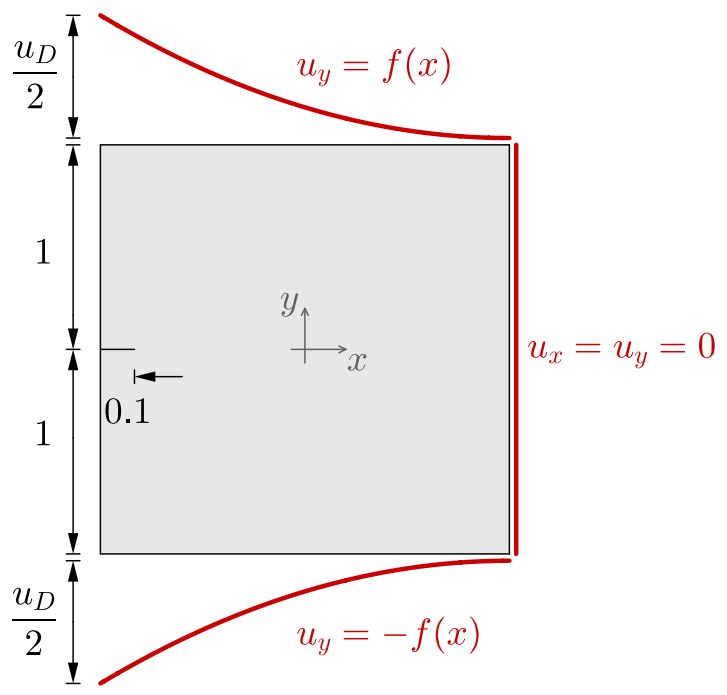

FIGURE 20 Branching test. Geometry and boundary conditions. Dimensions in mm.

\section{References}

1. Ambati M, Gerasimov T, De Lorenzis L. A review on phase-field models of brittle fracture and a new fast hybrid formulation. Comput Mech 2015; 55: 383-405.

2. Bourdin B, Francfort GA, Marigo JJ. Numerical experiments in revisited brittle fracture. J Mech Phys Solids 2000; 48: 797-826.

3. Miehe $\mathrm{C}$, Welschinger F, Hofacker M. Thermodynamically consistent phase-field models of fracture: variational principles and multi-field FE implementations. Int J Numer Methods Eng 2010; 83: 1273-1311.

4. Miehe C, Hofacker M, Welschinger F. A phase-field model for rate-independent crack propagation: robust algorithmic implementation based on operator splits. Comput Methods Appl Mech Eng 2010; 199: 2765-2778.

5. Borden MJ, Verhoosel CV, Scott MA, Hughes TJR, Landis CM. A phase-field description of dynamic brittle fracture. Comput Methods Appl Mech Eng 2012; 217-220: 77-95.

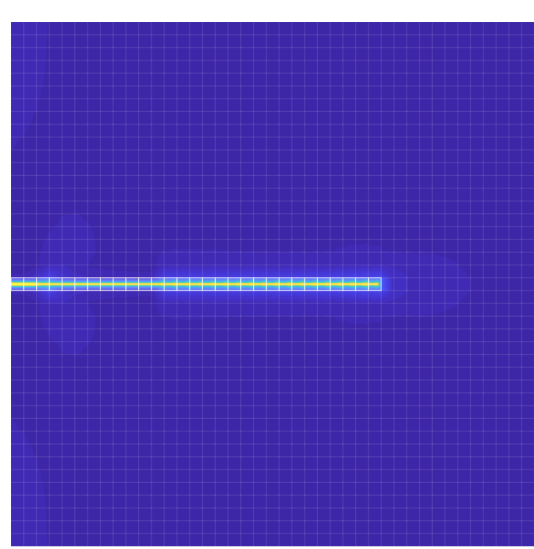

$u_{D}=0.03 \mathrm{~mm}$

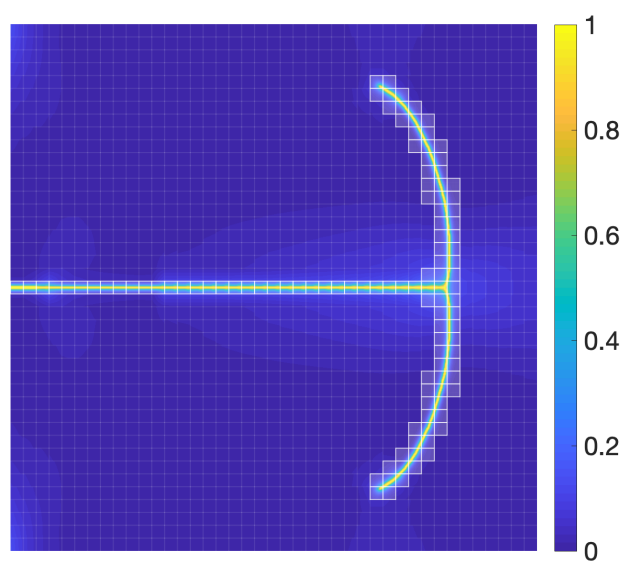

$u_{D}=0.07 \mathrm{~mm}$

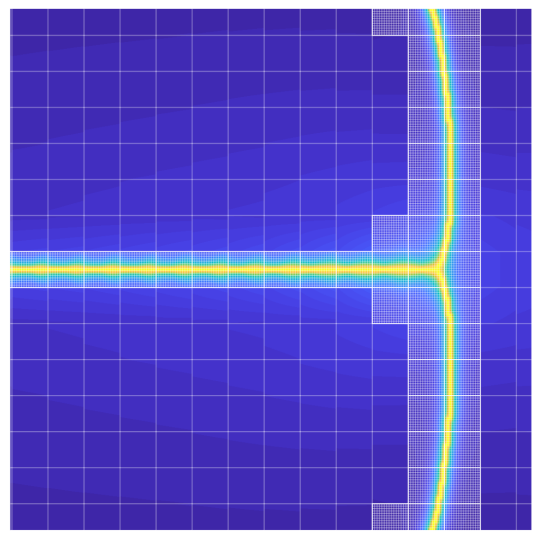

$u_{D}=0.07 \mathrm{~mm}($ zoom $)$

FIGURE 21 Branching test. Damage field at two different load steps, and zoom, for degree of approximation $p=1$ and refinement value $d_{\text {ref }}^{*}=0.2$. 
6. Nagaraja S, Elhaddad M, Ambati M, Kollmannsberger S, De Lorenzis L, Rank E. Phase-field modeling of brittle fracture with multi-level $h p$-FEM and the finite cell method. Comput Mech 2019; 6.

7. Patil RU, Mishra BK, Singh IV. A local moving extended phase field method (LMXPFM) for failure analysis of brittle fracture. Comput Methods Appl Mech Eng 2018; 342: 674-709.

8. Giovanardi B, Scotti A, Formaggia L. A hybrid XFEM-Phase field (Xfield) method for crack propagation in brittle elastic materials. Comput Methods Appl Mech Eng 2017; 320: 396-420.

9. Noii N, Aldakheel F, Wick T, Wriggers P. An Adaptive Global-Local Approach for Phase-Field Modeling of Anisotropic Brittle Fracture. Preprint, https://arxiv.org/pdf/1905.07519.pdf 2019.

10. Heister T, Wheeler MF, Wick T. A primal-dual active set method and predictor-corrector mesh adaptivity for computing fracture propagation using a phase-field. Comput Methods Appl Mech Eng 2015; 290: 466-495.

11. Hennig P, Müller S, Kästner M. Bézier extraction and adaptive refinement of truncated hierarchical NURBS. Comput Methods Appl Mech Eng 2016; 305: 316-339.

12. Hennig P, Ambati M, De Lorenzis L, Kästner M. Projection and transfer operators in adaptive isogeometric analysis with hierarchical B-splines. Comput Methods Appl Mech Eng 2018; 334: 313-336.

13. Muixí A, Fernández-Méndez S, Rodríguez-Ferran A. An HDG phase-field model for brittle fracture with adaptive refinement. https://www.youtube.com/watch?v=pjgxZz9vbLI, 2019.

14. Kirby RM, Sherwin SJ, Cockburn B. To CG or to HDG: A Comparative Study. J Sci Comput 2012; 51: $183-212$.

15. Yakovlev S, Moxey D, Kirby RM, Sherwin SJ. To CG or to HDG: A Comparative Study in 3D. J Sci Comput 2015; 67(1): $192-220$.

16. Giorgiani G, Modesto D, Fernández-Méndez S, Huerta A. High-order continuous and discontinuous Galerkin methods for wave problems. Int J Numer Methods Fluids 2013; 73(10): 883-903.

17. Paipuri M, Fernández-Méndez S, Tiago C. Comparison of high-order continuous and hybridizable discontinuous Galerkin methods in incompressible fluid flow problems. Mathematics and Computers in Simulation 2018; 153: 35-58.

18. Kronbichler M, Kormann K, Wall W. Fast matrix-free evaluation of hybridizable discontinuous Galerkin operators. Lecture Notes in Computational Science and Engineering 2019; 126: 581-589.

19. Francfort GA, Marigo JJ. Revisiting brittle fracture as an energy minimization problem. J Mech Phys Solids 1998; 46(8): 1319-1342.

20. Bourdin B, Francfort GA, Marigo JJ. The variational approach to fracture. J Elast 2008; 91(1-3): 5-148.

21. Wu JY, Nguyen VP, Nguyen CT, Sutula D, Bordas S, Sinaie S. Phase field modelling of fracture. Adv Appl Mech $2019 ; 53$.

22. Amor H, Marigo JJ, Maurini C. Regularized formulation of the variational brittle fracture with unilateral contact: numerical experiments. J Mech Phys Solids 2009; 57: 1209-1229.

23. Freddi F, Royer-Carfagni G, Stolarski HK. Regularized variational theories of fracture: A unified approach. J Mech Phys Solids 2010; 58: 1154-1174.

24. Soon SC, Cockburn B, Stolarski HK. A hybridizable discontinuous Galerkin method for linear elasticity. Int J Numer Methods Eng 2009; 80: 1058-1092.

25. Qiu W, Shen J, Shi K. An HDG method for linear elasticity with strong symmetric stresses. Math Comp 2018; 87(309): 69-93.

26. Sevilla R, Giacomini M, Karkoulias A, Huerta A. A super-convergent hybridisable discontinuous Galerkin method for linear elasticity. Int J Numer Methods Eng 2018; 116(2): 91-116. 
27. Cockburn B, Gopalakrishnan J, Lazarov R. Unified hybridization of discontinuous Galerkin, mixed and continuous Galerkin methods for second order elliptic problems. SIAM J Numer Anal 2009; 47: 1319-1365.

28. Fu G, Cockburn B, Stolarski H. Analysis of an HDG method for linear elasticity. Int J Numer Methods Eng 2015; 102: 551-575.

29. Cockburn B, Qiu W, Shi K. Conditions for superconvergence of HDG methods for second-order elliptic problems. Math Comp 2012; 81(279): 1327-1353.

30. Muixí A, Fernández-Méndez S, Rodríguez-Ferran A. A hybridizable discontinuous Galerkin phase-field model for brittle fracture.Reports@SCM 2018; 4(1):31-42.

31. Cervera M, Chiumenti M, Codina R. Mixed stabilized finite element methods in nonlinear solid mechanics Part I: Formulation. Comput Methods Appl Mech Eng. 2010; 199: 2559-2570.

32. Moës N, Dolbow J, Belytschko T. A finite element method for crack growth without remeshing. Int J Numer Methods Eng 1999; 46(1): 131-150.

33. Tamayo-Mas E, Rodríguez-Ferran A. A medial-axis-based model for propagating cracks in a regularised bulk. Int J Numer Methods Eng 2015; 101(7): 489-520.

34. Geelen RJ, Liu Y, Dolbow J, Rodríguez-Ferran A. An optimization-based phase-field method for continuous-discontinuous crack propagation. Int J Numer Methods Eng 2018; 116(1): 1-20. 